\title{
Host metabolite-cytokine correlation landscape in SARS-CoV-2 infection
}

Nan Xiao

Tsinghua University

Meng Nie

Tsinghua University

Huanhuan Pang

Tsinghua University

Bohong Wang

Tsinghua University

Jieli Hu

Chongqing Medical University

Xiangjun Meng

Tsinghua University

Ke Li

Chinese Academy of Medical Sciences \& Peking Union Medical College

Xiaorong Ran

Agilent Technologies (China),

Quanxin Long

Chongqing Medical University

Haijun Deng

Chongqing Medical University

Ni Tang ( $\square$ nitang@cqmu.edu.cn )

Chongqing Medical University

Ailong Huang ( $\nabla$ ahuang@cqmu.edu.cn )

Chongqing Medical University

Zeping Hu ( $\nabla$ zeping_hu@tsinghua.edu.cn )

Tsinghua University

\section{Research Article}

Keywords: COVID-19, SARS-COV-2, Metabolomics, Cytokine, Correlation analysis

Posted Date: October 19th, 2020 
DOI: https://doi.org/10.21203/rs.3.rs-91866/v1

License: (c) (1) This work is licensed under a Creative Commons Attribution 4.0 International License. Read Full License 
1 Host metabolite-cytokine correlation landscape in SARS-CoV-2 infection

2 Nan Xiao ${ }^{1,7}$, Meng $\mathrm{Ni}^{1,2,7}$, Huanhuan Pang ${ }^{1,2,7}$, Bohong Wang ${ }^{1,7}$, Jieli $\mathrm{Hu}^{3,7}$, Xiangjun

3 Meng ${ }^{1}, \mathrm{Ke} \mathrm{Li}^{4}$, Xiaorong Ran ${ }^{5}$, Quanxin Long ${ }^{3}$, Haijun Deng ${ }^{3}$, Ni Tang ${ }^{3 *}$, Ailong Huang ${ }^{3 *}$,

4 and Zeping $\mathrm{Hu}^{1,2,6,8 *}$

$5 \quad{ }^{1}$ School of Pharmaceutical Sciences, Tsinghua University, Beijing, 100084, China.

$6{ }^{2}$ Tsinghua-Peking Joint Center for Life Sciences, Tsinghua University, Beijing, 100084,

7 China.

$8 \quad{ }^{3}$ Key Laboratory of Molecular Biology for Infectious Diseases (Ministry of Education),

9 Chongqing Medical University, Chongqing, China.

$10{ }^{4} \mathrm{NHC}$ Key Laboratory of Biotechnology of Antibiotics, Institute of Medicinal 11 Biotechnology, Chinese Academy of Medical Sciences \& Peking Union Medical College, 12 Beijing, 100050, China

$13{ }^{5}$ Agilent Technologies (China), No. 3, Wang Jing Bei Lu, Chaoyang District, Beijing, 14 100102, China

$15{ }^{6}$ Beijing Frontier Research Center for Biological Structure, Tsinghua University, Beijing, 16100084 , China.

$17 \quad{ }^{7}$ These authors contributed equally

$18 \quad{ }^{8}$ Lead Contact

*Correspondence:

21 nitang@cqmu.edu.cn (N.T.), ahuang@cqmu.edu.cn (A.H.), zeping_hu@tsinghua.edu.cn 22 (Z.H.) 


\section{Abstract}

24 The systemic cytokine release syndrome (CRS) is a major cause of the multi-organ injury 25 and fatal outcome induced by SARS-CoV-2 infection in severe COVID-19 patients. It has

26 been well-known that metabolism plays a role in modulating the immune responses in

27 infectious diseases. Yet, how the host metabolism correlates with CRS in COVID-19

28 patients and how the perturbed metabolites affect the cytokine release remains unclear.

29 Here, we performed both metabolomics and cytokine/chemokine profiling on serum

30 samples from the same cohort of healthy controls, mild and severe COVID-19 patients and

31 delineated the global metabolic and immune response landscape along disease progression.

32 Intriguingly, the correlation analysis revealed the tight link between metabolites and

33 proinflammatory cytokines and chemokines, such as IL-6, M-CSF, IL-1 $\alpha$, IL-1 $\beta$, implying

34 the potential regulatory role of arginine metabolism, tryptophan metabolism, and purine

35 metabolism in hyperinflammation. Importantly, we demonstrated that targeting

36 metabolism markedly modulated the proinflammatory cytokines release by PBMCs

37 isolated from SARS-CoV-2-infected rhesus macaques ex vivo. Beyond providing a

38 comprehensive resource of metabolism and immunology data of SARS-CoV-2 infection,

39 our study showed that metabolic alterations can be potentially exploited to develop novel

40 strategy for the treatment of fatal CRS in COVID-19. 


\section{$41 \quad$ Introduction}

42 Coronavirus disease 2019 (COVID-19), caused by highly infectious severe acute

43 respiratory syndrome coronavirus 2 (SARS-CoV-2), has recently become global pandemic

$44{ }^{1}$, highlighting an urgent need for effective therapeutic strategies. SARS-CoV-2 infection

45 triggers immune response contributing to both virus clearance and acute respiratory distress

46 syndrome (ARDS) development ${ }^{2,3}$. The severe COVID-19 patients often experience

47 cytokine release syndrome (CRS) referred as "cytokine storm", which is characterized by

48 excessive proinflammatory cytokines production and leads to widespread damage, multiple

49 organ failure, and fatal clinical outcomes ${ }^{4-8}$. Emerging clinical trials uncover that

50 immunomodulatory drugs, such as the IL-6 receptor-blocking antibody tocilizumab and

51 JAK1/2 inhibitor ruxolitinib ${ }^{9-11}$, can dampen the hyperactive immune response, suggesting

52 cytokine-release blockade as a promising treatment option. Hence, identifying the key

53 factors driving CRS induced by SARS-CoV-2 infection is of utmost importance to provide

54 fresh insights for novel immunomodulatory therapies.

55 The association of metabolism and immunity has been reported since $1960 \mathrm{~s}^{12}$, and many

56 studies have shown that metabolic pathways can regulate the innate and adaptive host

57 responses to the infection of various viruses, such as human immunodeficiency virus (HIV)

$58{ }^{13}$, yellow fever virus ${ }^{14}$, severe fever with thrombocytopenia syndrome virus (SFTSV) ${ }^{15-}$

$59{ }^{17}$. Since the COVID-19 pandemic, studies exploring the immunological and metabolic

60 signatures in patients have been reported extensively 5,7,18,19. However, the host 
61 metabolism-immune response correlation and the potential modulatory role of the 62 perturbed metabolism in immune response upon SARS-CoV-2 infection remains largely 63 unknown. Here we performed both metabolomics and cytokine/chemokine profiling in 64 serum samples from the same cohort of COVID-19 patients and healthy controls. Strikingly, 65 correlation analysis of metabolites and cytokines revealed the potential metabolic 66 determinants of hyperinflammation to SARS-CoV-2 infection. Importantly, targeting 67 arginine metabolism, tryptophan metabolism, and purine metabolism by metabolite 68 supplementation or pharmacological inhibition modulated the ex vivo inflammatory 69 cytokine secretion by isolated peripheral blood mononuclear cells (PBMCs) derived from 70 SARS-CoV-2-infected rhesus macaques, indicating that metabolic interventions may be 71 potentially exploited as rational strategies to suppress CRS induced by COVID-19. 


\section{Study design and patient cohort}

74 To understand how the host metabolism correlates with CRS in COVID-19 patients and

75 how the perturbed metabolites may affect the cytokine release, we performed both

76 metabolomics and cytokine profiling on serum samples from the same COVID-19 patient

77 cohort. The cohort comprises of 17 healthy controls, 14 mild and 23 severe COVID-19

78 patients, as well as 7 mild patients with longitudinal follow-up time-points that occurred

79 4-36 days post symptom onset. We collected the clinical information and conducted

80 immunological and biochemical laboratory test as well as metabolomics and cytokine

81 profiling on the serum samples from these patients. To ascertain the potential modulatory

82 role of metabolism in cytokine secretion, we performed correlation analysis between

83 metabolites and cytokines, and validated the functional effect of metabolic intervention on

84 cytokine secretion ex vivo by isolated peripheral blood mononuclear cells (PBMCs) derived

85 from SARS-CoV2-infected rhesus macaque (Fig. 1a). The basic clinical features of the

86 cohort are detailed in Supplementary Table S1. Significant reductions in lymphocyte

87 count, and marked increase in C-reactive protein (CRP), alanine amino-transferase (ALT),

88 aspartate aminotransferase (AST), direct bilirubin (DBIL) and glucose were exhibited in

89 severe COVID-19 patients, which are consistent with previous findings ${ }^{1,20}$ (Extended

Data Fig. 1). 
92 To determine the metabolic perturbations associated with SARS-COV-2 infection, we 93 profiled the serum samples from 17 healthy controls and 44 COVID-19 patients by using 94 both targeted and untargeted metabolomics analyses (Extended Data Fig. 2a). Targeted 95 metabolomics analysis was performed on an ultra-high-performance liquid 96 chromatography-triple quadruple mass spectrometry system (UHPLC-MS/MS). A total of 97258 metabolites were monitored and 145 metabolites were reliably detected. Untargeted 98 metabolomics analysis was performed on an UHPLC quadruple TOF high resolution 99 MS/MS system. In total 6072 metabolite features were extracted from the raw data 100 acquired with positive and negative ionization modes and 1910 features were consistently 101 detected. Among these features, 161 metabolites were identified based on their accurate 102 mass, retention time, isotopic patterns and MS/MS spectra. By integrating the targeted and 103 untargeted metabolomics datasets, we identified in total 253 metabolites and observed 104 distinct metabolic profiles among healthy controls, mild and severe patients (Fig. 1b, 105 Extended Data Fig. 2b-d). Volcano plots highlighted 109 differential metabolites between 106 healthy controls and mild patients, and 98 differential metabolites between healthy controls 107 and severe patients, reflecting markedly dysregulated metabolic state of COVID-19 (Fig. 108 1c, d).

109 To characterize the dysregulated metabolic pathways in COVID-19 patients compared to 110 healthy controls, we performed pathway enrichment analyses and observed that primary 
111 bile acid biosynthesis, taurine and hypotaurine metabolism, amino acid metabolism, 112 nucleic acid metabolism, and citrate cycle (TCA cycle) were significantly perturbated in 113 both mild and severe patients (Fig. 1e). In contrast, metabolic pathways such as branched114 chain amino acid biosynthesis, glutathione metabolism, and pyruvate metabolism were 115 altered merely in mild patients, whereas thiamine metabolism, glutamine and glutamate 116 metabolism, and nitrogen metabolism were significantly changed specifically in severe 117 patients (Fig. 1e). Interestingly, the metabolites that displayed constant upward or 118 downward trend along disease progression were mostly associated with purine metabolism, 119 nicotinate and nicotinamide metabolism, tryptophan metabolism, TCA cycle and arginine 120 metabolism (Extended Data Fig. 2e). Intermediates in arginine metabolism have been 121 regarded as regulators of lymphocyte-suppression during immune response ${ }^{21}$. Our 122 previous work has demonstrated that arginine deficiency was associated with $\mathrm{T}$ cell 123 dysregulation in severe fever with thrombocytopenia syndrome virus (SFTSV) infection ${ }^{15}$. 124 Here we observed that glutamate and aspartic acid were upregulated, while glutamine and 125 citrulline were downregulated along disease progression (Extended Data Fig. 2f). 126 Succinate, an intermediate of TCA cycle that has been proved to be an innate immune 127 signaling molecule during inflammation in macrophage ${ }^{22}$, displayed a successive increase 128 along disease severity (Extended Data Fig. 2g). Moreover, the increase in kynurenine and 129 decline in tryptophan and serotonin suggested the enhanced activity of rate-limiting 130 enzyme indole 2,3-dioxygenase (IDO1) (Extended Data Fig. 2h), which was reported to 131 be a modulator of inflammation ${ }^{23,24}$. In addition, $\mathrm{NAD}^{+}$metabolism was found to be altered 
by host-pathogen interactions during innate and adaptive immune responses ${ }^{25}$, including

133 SARS-COV-2 infection ${ }^{26}$. Here the level of nicotinamide mononucleotide (NMN), a key

134 metabolite in the $\mathrm{NAD}^{+}$metabolism, decreased as the severity of COVID-19 increases

135 (Extended Data Fig. 2i). Taken together, our data delineated the global metabolic

136 alterations along the increase in the severity of COVID-19.

137 Cytokines secretion correlates with altered metabolism in COVID-19 patients

138 Extensive studies proved that metabolic pathways can control the innate and adaptive 139 immune response during viral infection ${ }^{13,27}$. To clarify the modulatory role of metabolism 140 in inflammation after SARS-CoV-2 infection, we assessed the cytokine and chemokine 141 levels in COVID-19 patients and healthy controls. Consistent with previous studies 142 reported ${ }^{5-8,28}$, COVID-19 patients presented marked elevation for most cytokines and 143 chemokines compared to healthy controls (Extended Data Fig. 3a). Particularly, the CRS144 related cytokines, including IL-6, IL-1 $\beta$, IL-10, IL-18 and IFN- $\gamma$, displayed progressive 145 increase along disease progression from healthy controls to mild and severe patients 146 (Extended Data Fig. 3b), highlighting the broad and strong inflammatory response in 147 severe COVID-19 patients.

148 In order to identify the metabolic alteration that might contribute to cytokine secretion in 149 serum of COVID-19 patients, we analyzed the correlation between the cytokines and 150 metabolites using linear regression after adjusting for age and gender. Systematic pathway 
151 analysis revealed a surprising number of significant correlations of cytokine alterations

152 with dysregulated metabolic pathways, mainly including amino acid metabolism, nucleic

153 acid metabolism, and energy metabolism (Fig. 2a). Moreover, most inflammatory

154 cytokines strongly correlated with metabolites involved in arginine metabolism, tryptophan

155 and $\mathrm{NAD}^{+}$metabolism, purine and pyrimidine metabolism, cysteine and methionine

156 metabolism, TCA cycle and primary bile acid metabolism (Fig. 2b-e). These observations

157 provide evidence that host metabolism broadly and highly correlated with cytokines linked

158 to CRS. Notably, the metabolites involved in arginine metabolism (i.e., arginine, glutamine,

159 guanidineacetic acid, creatinine, aspartic acid) displayed strongly negative correlation with

160 CRS-related cytokines (i.e., IL-6, M-CSF, IL-18, MCP-1, IL-12 p70, IFN- $\alpha 2$ ) (Fig. 2f). In

161 addition, purine metabolism (i.e., xanthine, guanosine, adenine) exhibited negative

162 correlation with CRS-related cytokines (i.e., IL-6, MCP-3, GM-CSF, IL-1 $\alpha$, IL-1 $\beta$ ) (Fig.

163 2g). Metabolites involved in tryptophan and $\mathrm{NAD}^{+}$metabolism, such as kynurenine and

164 NMN, showed positive correlation with MCP-3, M-CSF and IL-6. In contrast, tryptophan

165 and serotonin had negative correlation with IFN- $\gamma$ and MCP-1 (Fig. 2h). The correlations

166 between metabolites and cytokines in mild and severe patients suggest that the disturbances

167 in these metabolic pathways might potentially contribute to the hyperinflammation in

168 COVID-19.

169 Longitudinal metabolite-cytokine correlation in follow-up mild COVID-19 patients 
170 To further identify the dynamic correlations between metabolites and cytokines at 171 longitudinal stages after SARS-CoV-2 infection, we performed c-means clustering analysis

172 on both metabolite and cytokine data from the hospitalized mild patient with 4-36 follow173 up time-points. We identified four main clusters of longitudinal trajectories which 174 characterized distinct metabolic and immune signatures in these patients with acute 175 antibody responses to SARS-CoV-2 infection ${ }^{29}$ (Extended Data Fig. 4a, b). Molecules 176 enriched in cluster 1 increased at symptoms onset but gradually deceased during 177 hospitalization; molecules in cluster 2 exhibited a sharp decreased at symptoms onset and 178 sustained stable levels in later time-points. However, molecules in cluster 3 sustained 179 steady levels but presented a delayed elevation in the very late events; cluster 4 contained 180 molecules that elevated gradually and declined in late phases (Fig. 3a).

181 Three CRS-related cytokines including IL-6, IP-10 and M-CSF belonged to cluster 1 (Fig. 182 3b). Notably, the levels of IL-6, which is highly correlative to CRS ${ }^{30}$, decreased during the 183 first two weeks of symptom onset and remained at low level in later phases (Fig. 3b, 184 Extended Data Fig. 4c). The IFN- $\gamma$ inducible protein, IP-10/CXCL-10, is considered as a 185 member of CXC chemokine family with proinflammatory and severity-related properties 186 in COVID-19 ${ }^{8}$. The levels of IP-10 showed a sharp decline in the initial phase of treatment 187 and sustained at a relatively low levels during hospitalization (Fig. 3b, Extended Data Fig. 188 4c). Consistently, the myeloid cytokine M-CSF also showed a downward trend over the 189 course of mild disease similar to IP-10 (Fig. 3b, Extended Data Fig. 4c). In addition, 
191 showed decreased levels in mild patients compared to healthy controls and remained steady

192 levels during hospitalization (Fig. 3b, Extended Data Fig. 4c). However, proinflammatory 193 cytokines in cluster 3 (i.e., IL-17A and TNF- $\beta$ ) and cluster 4 (i.e., IL-1 $\alpha$, IL-1 $\beta$, IL-18 and 194 MCP-1) showed the upward trend along the follow-up time-points (Fig. 3b, Extended 195 Data Fig. 4c). These observations indicate that alleviation of inflammatory immune 196 responses may be accompanied with clinical recovery in hospitalized mild patients.

197 Interestingly, several cytokines enriched in cluster 4 were associated with suppression of 198 the inflammatory responses and viral replication, such as IL-10 and IFN- $\alpha 2$ (Fig. 3b, 199 Extended Data Fig. 4c). IL-10, which reportedly plays a role in antagonizing 200 inflammatory cell populations and suppressing immune hyperactivity ${ }^{31}$, continued to 201 elevate over time and maintained at high levels (Fig. 3b, Extended Data Fig. 4c). Also, 202 IFN- $\alpha 2$ is reported to play a crucial role in combating infection through inhibiting viral 203 replication and preventing viral entry into neighboring cells, thus used for treating several 204 viral infections, including hepatitis B and $\mathrm{C}^{32}$. We observed the steadily elevated and 205 sustained levels of IFN- $\alpha 2$ over hospitalization (Fig. 3b, Extended Data Fig. 4c). The 206 increased levels of IL-10 and IFN- $\alpha 2$ may reflect the presence of a negative feedback loop 207 to control the inflammatory response and virus infection. These data suggest that a 208 protective immune response may occur along with downward trend of proinflammatory 209 cytokines and clinical recovery in hospitalized mild patients ${ }^{33}$. 
210 We next characterized metabolites that were enriched in four clusters and specific 211 metabolite-cytokine correlations. Interestingly, metabolites associated with arginine 212 metabolism were enriched in cluster 2 and cluster 3 (Fig. 3c). We observed the upward 213 trend of arginine, ornithine, glutamate and proline, whereas the decrease in citrulline (Fig. 214 3d; Extended Data Fig. 5a, b). Arginine metabolism reportedly played a crucial role in 215 regulation of immune responses ${ }^{15,21}$. Correlation analysis showed that intermediates in 216 arginine metabolism highly correlated with proinflammatory cytokines including IL-6, M217 CSF and MIP-1 $\beta$ (Fig. 3e). Dysregulated tryptophan metabolism and $\mathrm{NAD}^{+}$metabolism 218 was evident by a marked alteration in associated metabolites exhibited four trajectories 219 (Fig. 3c). The increased metabolites (i.e., tryptophan, indole) and decreased metabolites 220 (i.e., kynurenine, kynurenic acid) reflected a hyperactivation of tryptophan-kynurenine 221 pathway (Fig. 3d, Extended data Fig. 5a, b), which plays an important role in modulating 222 inflammation ${ }^{23}$. Moreover, kynurenine positively correlated with proinflammatory 223 cytokines including IP-10, MCP-3 and M-CSF, whereas tryptophan negatively correlated 224 with MIP-1 $\alpha$, suggesting the regulatory role of tryptophan metabolism in inflammatory 225 response (Fig. 3e). Additionally, a large proportion of intermediates in purine metabolism 226 displayed the upward trend such as inosine and adenine (Fig. 3d, Extended data Fig. 5a, 227 b), and showed the negative correlation with most proinflammation cytokines (Fig. 3e). 228 Taken together, our data identify a panel of metabolite-cytokine correlation, which may 229 provide an unbiased way to determine the potential metabolic regulators of 230 proinflammatory cytokine secretion. 
232 Our data presented above delineated the strong correlation between cytokines and 233 metabolites, and identified metabolic pathways that are potentially crucial for 234 proinflammatory cytokine production. We next evaluated whether intervening arginine 235 metabolism, tryptophan metabolism and purine metabolism could regulate cytokine 236 induction. To this end, we isolated PBMCs from SARS-CoV-2-infected and mock-infected 237 rhesus macaques, and measured the cytokine concentrations after treatment with 238 metabolites or compounds interfering these key metabolic pathways (Fig. 4a). Interestingly, 239 we observed that supplementation of arginine markedly inhibited the SARS-CoV-2240 induced proinflammatory cytokines production by PBMCs, most of which linked to CRS 241 including IL-1 $\alpha$, IL-1 $\beta$, IL-2, IL-6, TNF- $\alpha$, GM-CSF, G-CSF and MIP-1 $\alpha$ (Fig. 4b, 242 Extended Data Fig. 6). Notably, the elevated level of IL-10, which is responsible for 243 inhibition of proinflammation cytokines production from macrophage and dendritic 244 cell (DC) populations ${ }^{31}$, was also suppressed (Fig. 4b). These observations suggest that 245 serum arginine metabolism may play a modulatory role in the hyperinflammation, thus 246 could be exploited as a potential therapeutic target for CRS in COVID-19.

247 The conversion of tryptophan into kynurenine in immune cells is finely regulated by the 248 enzyme indoleamine 2,3 dioxygenase 1 (IDO1), which is reportedly involved in regulating 249 hyperinflammatory responses ${ }^{24}$. The elevated ratio between circulating kynurenine and 250 tryptophan (Kyn/Trp) in patient's serum described above suggested an increased activity 
251 of IDO1. Addition of epacadostat, an IDO1 inhibitor, suppressed the SARS-CoV-2252 induced proinflammation cytokine release including IL-1 $\alpha$, IL-1 $\beta$, IL-6, TNF- $\alpha$, GM-CSF, 253 G-CSF, IL-17A and MIP-1 $\alpha$, which confirmed an essential role of tryptophan metabolism 254 in exaggerated cytokine release upon SARS-CoV-2 infection (Fig. 4c, Extended Data Fig. 255 7)). In addition, direct inhibition of purine metabolism with mycophenolic acid (MPA), 256 which blocks the rate-limiting enzyme inosine monophosphate dehydrogenase (IMPDH) 257 in de novo synthesis of guanosine nucleotides, significantly reduced levels of IL-10, IFN$258 \gamma$, IL-15, IL-12 p40, IL-17A and TNF- $\alpha$ induced by SARS-CoV-2 infection. However, a 259 profound increase in proinflammation cytokines of IL-6, GM-CSF, IL-1 $\alpha$ and IL-1 $\beta$ was 260 also observed, which suggests the exacerbated hyperinflammation upon interfering with 261 purine metabolism (Fig. 4d, Extended Data Fig. 8). Finally, we evaluated the effect of 262 interfering these metabolic pathways on the SARS-CoV-2 replication in PBMCs. Our 263 results showed that arginine supplementation, IDO1 or IMPDH inhibitors did not affect the 264 SARS-CoV-2 replication (Extended Data Fig. 9). Taken together, these data suggest that 265 targeting dysregulated host metabolism may serve as a viable approach to suppressing 266 SARS-CoV-2-induced inflammatory cytokine secretion. 


\section{Discussion}

268 CRS has been reported contribute to vascular damage, immunopathology, and adverse

269 clinical outcomes in COVID-19 patients 2,4,7. Hence, strategies to constrain the 270 proinflammation cytokines release are emerging as potential therapies for COVID-19 ${ }^{11,34}$.

271 Increasing studies have trialed strategies, including monoclonal antibodies targeting 272 inflammatory cytokines or small-molecule inhibiting the upstream or downstream 273 regulatory pathways, to dampen the inflammatory responses ${ }^{11,34}$. However, better 274 understanding the driving causes of cytokine storm and identifying potential multi275 cytokine blockers are still urgent need. Considering the previously reported high 276 correlation between metabolism and immune response ${ }^{13,17,27,35}$, and the key role of

277 metabolism in regulating cytokine release upon viral infection ${ }^{16,22}$, we speculated that the 278 metabolism and CRS may correlate well and correcting the dysregulated host metabolism 279 may suppress the SARS-CoV-2-induced CRS. We thus characterized the metabolic and 280 immune profiling in the same COVID-19 patient cohort, and our network correlation 281 analysis between circulating metabolite and cytokine levels revealed the potential 282 regulatory role of arginine metabolism, tryptophan metabolism, and purine metabolism in 283 proinflammatory responses.

284 The cytokine profiling of COVID-19 patients in our study provide further evidence that 285 most CRS-associated cytokines, such as IL-6, IL-1 $\beta$, IL-10, IL-18 and IFN- $\gamma$, are 286 dramatically elevated in severe patients. Conversely, the increased inflammatory responses 
experienced progressive reduction accompanying with clinical recovery in mild COVID-

28819 patients during hospitalization. These findings highlight the need for novel therapies to 289 block multiple cytokines linked to CRS. Emerging evidence suggests the key role of 290 metabolism in regulating cytokine secretion ${ }^{13,15,21}$. For example, choline uptake and 291 metabolism modulate the IL-1 $\beta$ and IL-18 production in stimulated macrophages ${ }^{36} . \alpha-$ 292 ketoglutarate-supplemented diet has been reported to induce IL-10 production, thus leading 293 to suppression of chronic inflammation and extension of life span ${ }^{37}$. Particularly, elevated 294 glucose levels in COVID-19 patients promote SARS-CoV-2 replication and cytokine 295 production in monocytes ${ }^{16}$. A very recent study suggests that the a high kynurenic acid to 296 kynurenine ratio is linked to immune responses and clinical outcomes in male COVID-19 297 patients ${ }^{38}$. In addition, Vitamin D deficiency associated with the uncontrolled cytokine 298 production and disease severity of COVID-19 ${ }^{39,40}$, emphasizing the need of Vitamin D 299 supplementation for COVID-19 treatment.

300 Our metabolomics data identified alterations of circulating metabolite levels in patient's 301 serum and determined dysregulated metabolic pathways upon SARS-CoV-2 infection. 302 Consistent with recent studies 18,41 , the metabolites that are associated with arginine 303 metabolism, tryptophan metabolism, TCA cycle as well as purine and pyrimidine 304 metabolism changed remarkedly. Interestingly, correlation network analysis between 305 metabolites and cytokines in mild and severe patients revealed that circulating cytokine 306 levels were highly correlated with arginine metabolism, tryptophan metabolism, nucleic 
307 acid metabolism and energy metabolism. Moreover, our time-series clustering analysis in 308 mild patients identified four distinct clusters of longitudinal trajectories delineating the 309 crosstalk between metabolism and inflammatory response. These results suggest 310 perturbation of metabolic pathways may partially contribute to the consequential CRS in 311 COVID-19.

312 Arginine is a conditionally essential amino acid for adult mammals and involves in immune 313 dysfunctions during viral infection ${ }^{15,21}$. Intriguingly, we observed that circulating levels of 314 arginine had a significant negative correlation with CRS-related proinflammatory 315 cytokines, and supplementation of arginine markedly inhibited the elevation of 316 proinflammatory cytokines upon SARS-CoV-2 infection, confirming its role as a potent 317 metabolic regulator. In addition, manipulation of tryptophan metabolism led to marked 318 decline in proinflammatory cytokines. Recent studies identified the important role of 319 IDO1-kynurenine/kynurenic acid-arylhydrocarbon receptors (AhRs) signaling in 320 inflammation and multiple organ injuries in SARS-CoV-2 infection ${ }^{38,42}$, which is 321 consistent with our findings. We also showed that inhibition of purine metabolism 322 exacerbated inflammatory response. However, the inhibition of pyrimidine biosynthesis 323 pathway reportedly arrested SARS-CoV-2 replication and suppressed inflammatory 324 cytokines production ${ }^{43}$. These results suggested the importance of the balance between 325 purine and pyrimidine metabolism in viral replication and immune response. Indeed, 326 combined agents targeting multiple pathogenic factors involved in the hyperinflammation 
327 is emerging as the way forward for supportive care for COVID-19 ${ }^{34}$. It is therefore possible 328 that cocktails of drugs targeting multiple metabolic pathways for global cytokine blockade 329 might constitute a new class of therapeutic strategy.

330 Due to the resource restriction, our study validated the effects of supplemented metabolites 331 or pharmacological inhibitors in regulating the CRS induced by SARS-CoV-2 infection 332 using the ex-vivo model of PBMCs isolated from the infected rhesus macaques. Although 333 the isolated PBMCs ex vivo models has been extensively used for the evaluation of 334 cytokine release ${ }^{44,45}$, it would have been ideal to perform such analyses in rhesus macaque 335 in vivo models. We tested the impacts of metabolism intervening on the immunological 336 responses in the heterogenous PBMCs. Yet, given that SARS-CoV-2 infection reduces 337 innate antiviral defenses while activates inflammatory cytokine release ${ }^{46-48}$, analyses on 338 sorted subpopulations of immune cells would help to more precisely understand the roles 339 of metabolism in regulating the release of specific cytokines with discriminating functions, 340 proinflammatory or suppressive, from different immune cell types.

341 In summary, our study performed a comprehensive metabolic and immune profiling in 342 COVID-19 patients and showed that reprogrammed host metabolism was tightly linked to 343 the burst of proinflammatory cytokines. Beyond providing a comprehensive resource of 344 metabolism and immunology data to support further investigation of COVID-19, our study 345 also uncovered new insights related to tight correlation between metabolism and cytokine 
346 release, and thereby provided potential therapeutic strategy for treatment of fatal CRS 347 induced by SARS-CoV-2 infection. 


\section{Reference}

349 1. Huang, C., et al. Clinical features of patients infected with 2019 novel

350 coronavirus in Wuhan, China. Lancet 395, 497-506 (2020).

351 2. Mehta, P., et al. COVID-19: consider cytokine storm syndromes and

352 immunosuppression. Lancet 395, 1033-1034 (2020).

353 3. Cao, X. COVID-19: immunopathology and its implications for therapy. Nat Rev

$354 \quad$ Immunol 20, 269-270 (2020).

355 4. Mangalmurti, N. \& Hunter, C.A. Cytokine Storms: Understanding COVID-19.

356 Immunity 53, 19-25 (2020).

357 5. Zhang, X., et al. Viral and host factors related to the clinical outcome of COVID-

358 19. Nature 583, 437-440 (2020).

359 6. Lucas, C., et al. Longitudinal analyses reveal immunological misfiring in severe

$360 \quad$ COVID-19. Nature 584, 463-469 (2020).

361 7. Del Valle, D.M., et al. An inflammatory cytokine signature predicts COVID-19

362 severity and survival. Nat Med (2020).

363 8. Laing, A.G., et al. A dynamic COVID-19 immune signature includes associations

364 with poor prognosis. Nat Med (2020).

365 9. Strohbehn, G.W., et al. COVIDOSE: Low-dose tocilizumab in the treatment of

366 Covid-19. medRxiv (2020).

367 10. Walz, L., et al. Janus Kinase-Inhibitor and Type I Interferon Ability to Produce

368 Favorable Clinical Outcomes in COVID-19 Patients: A Systematic Review and 369 Meta-Analysis. medRxiv (2020).

370 11. Luo, W., et al. Targeting JAK-STAT Signaling to Control Cytokine Release

371 Syndrome in COVID-19. Trends Pharmacol Sci 41, 531-543 (2020).

372 12. Oren, R., Farnham, A.E., Saito, K., Milofsky, E. \& Karnovsky, M.L. Metabolic

373 patterns in three types of phagocytizing cells. J Cell Biol 17, 487-501 (1963).

374 13. Saez-Cirion, A. \& Sereti, I. Immunometabolism and HIV-1 pathogenesis: food for 
thought. Nat Rev Immunol (2020).

376 14. Chan, K.R., et al. Metabolic perturbations and cellular stress underpin susceptibility to symptomatic live-attenuated yellow fever infection. Nat Med 25,

379 15. Li, X.K., et al. Arginine deficiency is involved in thrombocytopenia and

16. Codo, A.C., et al. Elevated Glucose Levels Favor SARS-CoV-2 Infection and Monocyte Response through a HIF-1alpha/Glycolysis-Dependent Axis. Cell Metab 32, 437-446 e435 (2020).

17. Sica, A., et al. Immunometabolic Status of COVID-19 Cancer Patients. Physiol Rev 100, 1839-1850 (2020).

18. Shen, B., et al. Proteomic and Metabolomic Characterization of COVID-19 Patient Sera. Cell 182, 59-72 e15 (2020).

19. Song, J.W., et al. Omics-Driven Systems Interrogation of Metabolic Dysregulation in COVID-19 Pathogenesis. Cell Metab 32, 188-202 e185 (2020).

20. Guan, W.J., et al. Clinical Characteristics of Coronavirus Disease 2019 in China. N Engl J Med 382, 1708-1720 (2020).

21. Bronte, V. \& Zanovello, P. Regulation of immune responses by L-arginine metabolism. Nat Rev Immunol 5, 641-654 (2005).

22. Tannahill, G.M., et al. Succinate is an inflammatory signal that induces IL-1beta through HIF-1alpha. Nature 496, 238-242 (2013).

23. Cervenka, I., Agudelo, L.Z. \& Ruas, J.L. Kynurenines: Tryptophan's metabolites in exercise, inflammation, and mental health. Science 357(2017).

24. Belladonna, M.L. \& Orabona, C. Potential Benefits of Tryptophan Metabolism to the Efficacy of Tocilizumab in COVID-19. Front Pharmacol 11, 959 (2020).

25. Mesquita, I., et al. Exploring NAD+ metabolism in host-pathogen interactions. 
Cell Mol Life Sci 73, 1225-1236 (2016).

403 26. Heer, C.D., et al. Coronavirus and PARP expression dysregulate the NAD Metabolome: a potentially actionable component of innate immunity. bioRxiv (2020).

27. Beisel, W.R. Metabolic response to infection. Annu Rev Med 26, 9-20 (1975).

28. Wilk, A.J., et al. A single-cell atlas of the peripheral immune response in patients with severe COVID-19. Nat Med 26, 1070-1076 (2020).

29. Long, Q.X., et al. Antibody responses to SARS-CoV-2 in patients with COVID19. Nat Med 26, 845-848 (2020).

30. Moore, J.B. \& June, C.H. Cytokine release syndrome in severe COVID-19. Science 368, 473-474 (2020).

31. Couper, K.N., Blount, D.G. \& Riley, E.M. IL-10: the master regulator of immunity to infection. J Immunol 180, 5771-5777 (2008).

32. Chen, J., et al. Functional Comparison of IFN-alpha Subtypes Reveals Potent HBV Suppression by a Concerted Action of IFN-alpha and -gamma Signaling. Hepatology (2020).

33. Zhou, Q., et al. Interferon-alpha2b Treatment for COVID-19. Front Immunol 11, 1061 (2020).

34. Harrison, C. Focus shifts to antibody cocktails for COVID-19 cytokine storm. Nat Biotechnol 38, 905-908 (2020).

35. Wang, A., Luan, H.H. \& Medzhitov, R. An evolutionary perspective on immunometabolism. Science 363(2019).

36. Sanchez-Lopez, E., et al. Choline Uptake and Metabolism Modulate Macrophage IL-1beta and IL-18 Production. Cell Metab 29, 1350-1362 e1357 (2019).

37. Asadi Shahmirzadi, A., et al. Alpha-Ketoglutarate, an Endogenous Metabolite, Extends Lifespan and Compresses Morbidity in Aging Mice. Cell Metab 32, 447 456 e446 (2020). 
38. Cai, Y., et al. Kynurenic acid underlies sex-specific immune responses to COVID19. medRxiv, 2020.2009.2006.20189159 (2020).

39. Mardani, R., et al. Association of vitamin D with the modulation of the disease severity in COVID-19. Virus Res, 198148 (2020).

40. Daneshkhah, A., et al. Evidence for possible association of vitamin D status with cytokine storm and unregulated inflammation in COVID-19 patients. Aging Clin Exp Res (2020).

41. Thomas, T., et al. COVID-19 infection alters kynurenine and fatty acid metabolism, correlating with IL-6 levels and renal status. JCI Insight 5(2020).

42. Turski, W.A., Wnorowski, A., Turski, G.N., Turski, C.A. \& Turski, L. AhR and IDO1 in pathogenesis of Covid-19 and the "Systemic AhR Activation Syndrome:" Translational review and therapeutic perspectives. Restor Neurol Neurosci (2020).

43. Luban, J., et al. The DHODH Inhibitor PTC299 Arrests SARS-CoV-2 Replication and Suppresses Induction of Inflammatory Cytokines. bioRxiv, 2020.2008.2005.238394 (2020).

44. Wang, Q., et al. O-GlcNAc transferase promotes influenza A virus-induced cytokine storm by targeting interferon regulatory factor-5. Sci Adv 6, eaaz7086 (2020).

45. Lee, A.C.Y., et al. Avian influenza virus A H7N9 infects multiple mononuclear cell types in peripheral blood and induces dysregulated cytokine responses and apoptosis in infected monocytes. J Gen Virol 98, 922-934 (2017).

46. Hadjadj, J., et al. Impaired type I interferon activity and inflammatory responses in severe COVID-19 patients. Science 369, 718-724 (2020).

47. Tay, M.Z., Poh, C.M., Renia, L., MacAry, P.A. \& Ng, L.F.P. The trinity of COVID-19: immunity, inflammation and intervention. Nat Rev Immunol 20, 363 $374(2020)$.

48. Blanco-Melo, D., et al. Imbalanced Host Response to SARS-CoV-2 Drives 

Development of COVID-19. Cell 181, 1036-1045 e1039 (2020).

457 49. Huang, F., et al. Inosine Monophosphate Dehydrogenase Dependence in a Subset 458 of Small Cell Lung Cancers. Cell Metab 28, 369-382 e365 (2018). 


\section{Patients and samples}

461 A total of 44 COVID-19 patients and 17 healthy controls were enrolled in this study. Cross462 sectional serum samples from 37 COVID-19 patients were collected from Chongqing 463 Three Gorges Central Hospital, Chongqing Public Health Medical Center and Yongchuan 464 Hospital Affiliated to Chongqing Medical University. Sequential serum samples from 7 465 mild patients were collected from Yongchuan Hospital Affiliated to Chongqing Medical 466 University with intervals of 3 days. Serum samples from 17 healthy controls were collected 467 from The Second and Third Affiliated Hospital of Chongqing Medical University. Patients 468 were confirmed to be infected with SARS-CoV-2 by RT-PCR assays (DAAN Gene) on 469 nasal and pharyngeal swab specimens. Briefly, two target genes, including open reading 470 framelab (ORF1ab) and nucleocapsid protein $(\mathrm{N})$, were simultaneously amplified and 471 tested during RT-PCR. Primers of RT-PCR testing for SARS-CoV-2 were designed 472 according to the recommendation by the Chinese CDC (ORF1ab-forward: 473 CCCTGTGGGTTTTACACTTAA, ORF1ab-reverse: ACGATTGTGCATCAGCTGA, 474 ORF1ab-probe: 5'-CCGTCTGCGGTATGTGGAAAGGTTATGG-3' (FAM dye labeled);

475 N-forward: GGGGAACTTCTCCTGCTAGAAT, N-reverse: 476 CAGACATTTTGCTCTCAAGCTG， N-probe: 5'-TTGCTGCTGCTTGACAGATT-3' 477 (VIC dye labeled)). PCR cycling: $50^{\circ} \mathrm{C}$ for $15 \mathrm{~min}, 95^{\circ} \mathrm{C}$ for $15 \mathrm{~min}, 45$ cycles containing 

than 40 were defined as positive and negative, respectively, for both genes.

\section{Detection of IgG and IgM against SARS-CoV-2}

481 All serum samples were inactivated at $56^{\circ} \mathrm{C}$ for $30 \mathrm{~min}$ and stored at $-20{ }^{\circ} \mathrm{C}$ before testing. 482 IgG and IgM against SARS-CoV-2 in plasma samples were tested using magnetic 483 chemiluminescence enzyme immunoassay kits supplied by Bioscience Co. (approved by 484 the China National Medical Products Administration; approval numbers 20203400183(IgG) 485 and 20203400182(IgM)), according to the manufacturer's instructions. Antibody levels are 486 presented as the measured chemiluminescence values divided by the cutoff $(\mathrm{S} / \mathrm{CO})$.

\section{Serum sample cytokine measurement}

488 Concentrations of 48 cytokines and chemokines in each serum sample were measured 489 using the Bio-Plex Human Cytokine Screening Panel (48-Plex no. 12007283, Bio-Rad) on 490 a Luminex 200 (Luminex Multiplexing Instrument, Merck Millipore) following the 491 manufacturer's instructions.

\section{Clinical data collection}

493 Epidemiologic, demographic, clinical presentations, laboratory tests, treatment and 494 outcome data were collected from inpatient medical records. Laboratory data collected for 495 each patient included complete blood count, coagulation profile, serum biochemical tests 
496 (including renal and liver function, electrolytes, lactate dehydrogenase and creatine kinase), 497 serum ferritin and biomarkers of infection.

498

499

500

501

502

503

504

505

506

507

508

509

510

511

\section{Clinical Definitions}

Clinical classification was defined based on the COVID-19 Treatment Guidelines (National Health Commission of the People's Republic of China). A confirmed case of SARS-CoV-2 infection was defined as an individual with nasopharyngeal swabs positive for SARS-CoV-2 nucleic acid by RT-PCR as described above. Severe COVID-19 cases were whom meeting any of the following criteria: 1$)$ respiratory distress $(\geq 30$ times/minutes), 2) the oxygen saturation $\leq 93 \%$ at rest, 3) the arterial partial pressure of oxygen $(\mathrm{PaO} 2) /$ the fraction of inspired oxygen $(\mathrm{FiO} 2) \leq 300 \mathrm{mmHg}$. Mild patients were defined as COVID-19 patients with symptoms but could not be classified as severe. Symptoms onset date was defined as the date on which symptoms first bean. Symptoms included fever, fatigue, dry cough, inappetence, myalgia, dyspnea, expectoration, pharyngalgia, diarrhea, nausea, dizziness, headache, abdominal pain, chill, rhinorrhea, chest stuffiness or nasal congestion.

\section{Ethical approval}

512 The study was approved by the Ethics Commission of Chongqing Medical University (ref. 513 no. 2020003). Written informed consent was waived by the Ethics Commission of the 514 designated hospitals for emerging infectious diseases. 


\section{Sample preparation for metabolomics}

516 Human serum samples, $20 \mu \mathrm{L}$ each, were heated at $56^{\circ} \mathrm{C}$ for $30 \mathrm{~min}$ followed by adding

$51760 \mu \mathrm{L}$ ethanol to inactive SARS-COV-2 virus. The suspension was evaporated to dryness

518 using a SpeedVac concentrator (Thermo Scientific). Metabolites from the serum pellet

519 were extracted with $540 \mu \mathrm{L} 80 \%$ methanol in water, followed by vigorous vortex and

520 cooled centrifuge at $4{ }^{\circ} \mathrm{C}$. Then, equal aliquots of the supernatant from each sample $(20 \mu \mathrm{L})$

521 were pooled together to make the quality control (QC) samples. The remaining supernatant

522 was divided into two fractions, one for targeted metabolomics and the other for untargeted

523 metabolomics analysis. All the samples were evaporated to dryness.

\section{Targeted metabolomics}

525 For targeted metabolomics, dried metabolites were reconstituted in LC-MS grade water

526 with $0.03 \%$ formic acid, vortex-mixed and centrifuged at $4^{\circ} \mathrm{C}$ for $15 \mathrm{~min}$ to remove debris.

527 Samples were randomized and blinded before analyzing by LC-MS/MS.

528 Chromatographic separation was performed on a Nexera Ultra High-Performance Liquid

529 Chromatograph (UHPLC) system (Shimadzu), with a RP-UPLC column (HSS T3, $2.1 \mathrm{~mm}$

$530 \times 150 \mathrm{~mm}, 1.8 \mu \mathrm{m}$, Waters) and the following gradient: $0-3 \mathrm{~min} 99 \% \mathrm{~A} ; 3-15 \mathrm{~min} 99-1 \%$

531 A; $15-17 \min 1 \%$ A; $17-17.1 \min 1-99 \%$ A; $17.1-20$ min 99\% A. Mobile Phase A was 0.03\%

532 formic acid in water. Mobile Phase B was $0.03 \%$ formic acid in acetonitrile. The flow rate

533 was $0.25 \mathrm{~mL} \cdot \mathrm{min}^{-1}$, the column was at $35^{\circ} \mathrm{C}$ and the autosampler was at $4^{\circ} \mathrm{C}$. Mass data 
534 acquisition was performed using an AB QTRAP 6500+ triple quadrupole mass 535 spectrometer (SCIEX, Framingham, MA) in multiple reaction monitoring (MRM) mode 536 for the detection of 258 unique endogenous water-soluble metabolites as previously 537 described, with some modifications ${ }^{15,49}$. Chromatogram review and peak area integration 538 were performed using MultiQuant 3.0.2 (SCIEX, Framingham, MA).

539 Untargeted metabolomics

540 For untargeted metabolomics, dried samples were reconstituted in acetonitrile/water 541 mixture $(\mathrm{v} / \mathrm{v}, 1: 1)$. After vortex, samples were centrifuged at $4^{\circ} \mathrm{C}$ for $15 \mathrm{~min}$ to remove 542 debris. Samples were randomized and blinded before LC-MS/MS analysis.

543 Chromatographic separation was performed on an Agilent 1290 infinity II LC system, with 544 an Agilent Eclipse Plus C18 column $(2.1 \mathrm{~mm} \times 100 \mathrm{~mm}, 1.8 \mu \mathrm{m})$. The gradient was set as 545 follows: $0-2 \min 95 \% \mathrm{~A} ; 2-20 \min 95-0 \% \mathrm{~A} ; 20-25 \mathrm{~min} 0 \% \mathrm{~A}$; post-run time for 546 equilibration, $5 \mathrm{~min}$ in $95 \% \mathrm{~A}$. Water containing $0.1 \%$ formic acid and acetonitrile 547 containing $0.1 \%$ formic acid acted as the mobile phase $\mathrm{A}$ and $\mathrm{B}$ in the positive-ion mode 548 of mass spectrometry analysis. While in the negative-ion mode of mass spectrometry 549 analysis, the $0.1 \%$ formic acid was replaced with $1 \mathrm{mM}$ ammonium fluoride. The flow rate 550 was set as $0.3 \mathrm{~mL} \cdot \mathrm{min}^{-1}$ and the temperatures of the column and autosampler were set as $55140^{\circ} \mathrm{C}$ and $4^{\circ} \mathrm{C}$ separately. The data acquisition was performed on a 6546 Q-TOF mass 552 spectrometry equipped with a dual electrospray (ESI) ion source (Agilent Technologies, 
553 Santa Clara, CA). The optimized ESI Q-TOF parameters were set as follows. The 554 temperature and flow rate of sheath gas were $350^{\circ} \mathrm{C}$ and $11 \mathrm{~L} \cdot \mathrm{min}^{-1}$. The voltages of 555 capillary, fragmentor and skimmer were set as $4000 \mathrm{~V}, 140 \mathrm{~V}$ and $65 \mathrm{~V}$, respectively. The 556 spectra were internally mass calibrated in real time by continuous infusion of a reference 557 mass solution using an isocratic pump connected to a dual sprayer feeding into an ESI 558 source. MassHunter Acquisition software (Agilent Technologies, Santa Clara, CA) was 559 employed to perform data acquisition.

560 The Agilent Masshunter Workstation (Profinder software, version 10.0) was firstly utilized 561 to filter the candidate metabolites. The metabolites were identified based on their accurate 562 mass, retention time, isotopic patterns. And the qualitative analysis software (version 10.0)

563 was employed to check the MS/MS spectra of the identified metabolites to avoid false

564 results. Further, to expand the qualitative coverage of specific metabolic pathways, the 565 Agilent Pathway to PCDL software was employed to perform targeted extraction of 566 metabolites from the raw data. The peak areas of the identified metabolites and the other 567 metabolite features which can be consistently detected from $80 \%$ samples were achieved 568 from the Profinder software.

\section{Normalization and integration of targeted and untargeted metabolomics data}

570 For both targeted and untargeted metabolomics, quality control (QC) samples composed 571 of an equal aliquot of all test samples were prepared and inserted in an interval of ten test 
572 samples to monitor the stability of instrument and normalize the variations during the run.

573 This served as an additional quality control measure of analytical performance and a 574 reference for normalizing raw metabolomic data across samples.

575 To remove potential inter-batch variations, the mean peak area of each metabolite from all 576 the QC samples in a given batch $\left(\mathrm{QC}_{\mathrm{all}}\right)$, as well as the mean peak area of each metabolite 577 from the QC samples that are the most adjacent to a given group of test samples (QC $\left.\mathrm{Cdj}_{\mathrm{aj}}\right)$ 578 were first calculated. The ratio between these two mean peak areas for each metabolite was 579 computed by dividing the same $\mathrm{QC}_{\text {all }}$ by each $\mathrm{QC}_{\mathrm{adj}}$ and used as the normalization factor 580 for each given group of test samples. The peak area of each metabolite from each test 581 sample were normalized by multiplying their corresponding normalization ratio to obtain 582 the normalized peak areas. In addition, to effectively correct the sample to sample variation 583 in biomass that may contribute to systematic differences in metabolite abundance detected 584 by LC-MS, we generated the scaled data by comparing the normalized peak area of each 585 metabolite to the sum of the normalized peak area from all the detected (for targeted 586 metabolomics) or identified metabolites (for untargeted metabolomics) in that given 587 sample.

588 Our validation analyses suggested that these normalization and scaling steps could 589 effectively correct both the inter-sample artificial differences in sample biomass and inter590 batch systematic variations in detected metabolite abundance. 
591 For metabolites that were detected in both targeted and untargeted methods, data from 592 targeted method were used for the subsequent analysis, except for the data of carnitine 593 metabolites which are from untargeted methods. The final metabolomic data matrix (102 594 rows of patients/controls and 253 columns of metabolites) used for the downstream 595 analyses or modeling is included in Table $\mathbf{S 2 .}$

596 Virus preparation

597 Viral stocks of SARS-CoV-2 were obtained from the Center of Diseases Control, 598 Guangdong Province China. Virus were amplified on Vero E6 cells and concentrated by 599 ultrafilter system via $300 \mathrm{kDa}$ module (Millipore). Vero E6 cells were cultured in Roswell 600 Park Memorial Institute (RPMI) 1640 medium supplemented with 10\% fetal bovine 601 serum (FBS).

602 Isolation of PBMCs from monkey peripheral blood

603 Peripheral blood mononuclear cells (PBMCs) were isolated from monkey peripheral blood 604 using Ficoll-PaqueTM (Sigma-Aldrich). Peripheral blood sample (4 mL) from each mock605 infected and SARS-CoV-2-infected Rhesus Macaques was drawn into vacutainer tubes. 606 The ficoll density gradient centrifugation method was used to separate the PBMCs. We 607 diluted the blood with $1 \times$ phosphate-buffered saline (PBS) 1:1 and then transferred it to the 608 ficoll tube. After centrifugation at $1000 \times \mathrm{g}$ for $20 \mathrm{~min}$, the buffy coat of PBMCs was pooled 
611 Antiviral assays

612 PBMCs $\left(2 \times 10^{4}\right.$ cells / well $)$ isolated from mock-infected rhesus macaques were seeded in 613 24-well plates and infected with mock or SARS-CoV-2 at MOI 0.05. Virus was added 614 together with drugs and incubated in RPMI 1640 medium supplemented with 10\% FBS 615 with different drugs. Viral loads of SARS-CoV-2 in PBMCs were assessed $24 \mathrm{~h}$ after 616 infection.

617 Viral RNA measurements

618 SARS-CoV-2 RNA from PBMCs was isolated using the Direct-zol $^{\mathrm{TM}}$ RNA 619 MiniPrep (Zymo Research Corp) according to the manufacturer's instructions. Briefly, 50 $620 \mu \mathrm{L}$ DNase/RNase-Free Water was used to elute RNA. Real time RT-PCR was used to 621 quantify viral genome in samples using TaqMan Fast Virus One-Step Master Mix (Thermo 622 Fisher) and purified viral RNA of SARS-CoV-2 as a standard curve, performed on CFX384 623 Touch Real-Time PCR Detection System (Bio-Rad). Conditions for RT-PCR were used as 624 follows: $25^{\circ} \mathrm{C}$ for $2 \mathrm{~min}, 50^{\circ} \mathrm{C}$ for $15 \mathrm{~min}, 95^{\circ} \mathrm{C}$ for $2 \mathrm{~min}$, then 40 cycles at $95^{\circ} \mathrm{C} 5 \mathrm{sec}$ 625 and $60^{\circ} \mathrm{C} 31 \mathrm{sec}$. Primers and probe, specific for SARS-CoV-2 NP gene was synthesized 626 according to sequences reported by China Centers for Disease Control (CDC), Target-2-F: 
Measurement of cytokines production by PBMCs

PBMCs $\left(1 \times 10^{6}\right.$ cells/well $)$ isolated from mock-infected and SARS-CoV-2-infected

Rhesus Macaques were seeded in 96-well plates and treated with different drugs. For cytokine measurement of PBMCs culture supernatant samples, MILLIPLEX MAP Nonprotocol, which was performed on Bio-plex machine. Inflammatory cytokines in this panel included IL-1 $\beta$, IL-4, IL-5, IL-6, IL-8/CXCL8, G-CSF, GM-CSF, IFN- $\gamma$, IL-1RA, IL-2, IL$1 \alpha /$ CCL3, sCD40L, TGF- $\alpha$, TNF- $\alpha$, VEGF and IL-18.

\section{Animals and experimental procedures}

For SARS-CoV-2 virus infections, we inoculated rhesus macaques with total $5 \mathrm{~mL}$ of $10^{6}$

$641 \mathrm{pfu} / \mathrm{mL}$ SARS-CoV-2 intratracheally $(2.5 \mathrm{~mL})$ and intranasally $(2.5 \mathrm{~mL})$. All animal

642 experiments were performed according to protocols approved by the Institutional Animal

643 Care and Use Committee of Institute of Medical Biology, Chinese Academy of Medical

644 Science, and performed in the ABSL-4 facility of Kunming National High-level Biosafety

645 Primate Research Center, Yunnan China. 
647 PLS-DA was performed on normalized metabolomics data using SIMCA-P

648 software (version 14.1, Umetrics, Umea, Sweden) and unit variance (UV) scaling was

649 utilized before multivariate analysis.

651 t-SNE scatterplots were generated of log10-transformed normalized metabolomics data 652 using R Rtsne (v0.15) package with perpiexity of 5 and theta of 0.01 .

\section{Longitudinal trajectory analysis of serum metabolites and cytokines}

654 To estimate serum cytokine and metabolite longitudinal trajectories of follow-up mild

655 COVID-19 patients, generalized addictive model (GAM) adjusted for age and gender was

656 fitted for each cytokine and metabolite. GAM was performed by R mgcv (v1.8-31) package

657 with default parameters.

658 Mann-Whitney U tests and Benjamini-Hochberg multiple comparison test were carried out 659 at each time point to identify significant altered serum cytokines and metabolites compared 660 with healthy controls (base lines). Cytokines and metabolites with FDR $<0.05$ were 661 considered significant. Based on the relative abundance of significant metabolites and 662 cytokines after z-score scaled, fuzzy c-mean clustering was performed using R 663 Mfuzz (v2.44.0) package, and parameter $\mathrm{m}$ was set to 1.5. 
665 Linear regressions models adjusted for gender and age were used to estimate correlations

666 between serum metabolites and cytokines, as log10-transformed, in mild and severe

667 patients or follow-up patients. Linear regressions models conducted in $\mathrm{R}$ using the $\operatorname{lm}$ base

668 function. For mild and severe patients, cytokines and metabolites which were significantly

669 altered in severe patients were applied to linear regression models. For follow-up patients,

670 metabolites and core CRS-related cytokines involved in four clusters were applied to linear

671 regression models. $P$ values were corrected using Benjamini-Hochberg multiple

672 comparison test and correlations with $\mathrm{FDR}<0.05$ were considered significant and used for

673 subsequent analysis.

674 Then, based on the significant cytokine-metabolite correlations in serum of mild and severe

675 patients, weighted, undirected correlation networks were built by R igraph (v1.2.5) package

676 and clusters were determined based on fast greedy modularity optimization algorithm.

677 Molecule types and correlation directions were color coding.

\section{KEGG pathway analysis}

679 Serum metabolites identified by targeted and untargeted metabolomics were integrated for 680 KEGG pathway analysis. KEGG metabolic pathways and involved metabolites were 681 downloaded through KEGG API (https://www.kegg.jp/kegg/rest/keggapi.html). 682 Significant enriched KEGG pathways based on given serum metabolites were determined 
683 by R clusterProfiler (v3.12.0) package with Benjamini-Hochberg multiple comparison test. 684 For significantly altered serum metabolites in mild and severe patients, pathways with FDR $685<0.1$ were considered significant. For metabolites significantly associated with cytokines 686 in mild and severe patients, pathways with FDR $<0.1$ and enriched for at least 3 cytokines 687 were considered enriched. For serum metabolites in each trajectory cluster in follow-up 688 patients, pathways with FDR $<0.05$ were considered enriched.

689 For metabolites with progressive change in mild and severe patients, metabolites which 690 increased or decreased in mild patients (compared with healthy controls) and severe 691 patients (compared with mild patients) and showed significance in severe patients were 692 considered consistently altered. Metabolic pathways with FDR $<0.1$ were considered 693 significantly enriched.

\section{Statistical analysis}

695 Quantification methods and statistical analysis methods for metabolite and cytokine 696 analyses were mainly described and referenced in the respective Method Details 697 subsections.

698 Additionally, Serum metabolites and cytokines with significant altered abundance were 699 identified by Mann-Whitney U tests and Benjamini-Hochberg multiple comparison test, 700 which were performed by R stat (v3.6.0) package. Serum cytokines and metabolites with 701 FDR $<0.05$ were considered significant and used for subsequent analysis. Statistical 
702 significance of cytokine and viral RNA abundance in cell culture supernatant between 703 different conditions were considered using one-way ANOVA followed by Benjamini704 Hochberg multiple comparison test, which were performed by GraphPad prism (v8.2.1).

705 Acknowledgments: We thank W.Z., X.H., Q.D., X.L. and members of the Hu laboratory 706 for critiquing the manuscript. Z.H. is supported by grants from National Key R\&D Program 707 of China (2019YFA0802100, 2019YFA0802100-02), National Natural Science 708 Foundation of China (81973355), National Science and Technology Major Project for 709 "Significant New Drugs Development" (2017ZX09304015), Tsinghua University 710 (53332200517), Tsinghua-Peking Joint Center for Life Sciences, and Beijing Frontier 711 Research Center for Biological Structure.

712 Author contributions: N.X., M.N. and Z.H. conceived the project, designed the study and 713 wrote the paper. N.X. designed and performed the metabolite-cytokine correlation analyses 714 and interpreted the results. M.N. and K.L. designed and performed the experiments related 715 to PBMCs derived from rhesus macaques. H.P. and B.W. performed the metabolomics 716 experiments. X.M. and X.R. assisted in the metabolomics experiments. T.J., P.Z., W.J., 717 Q.Y., and C.Z. provided the clinical samples and information. J.H., Q.L. and N.T. 718 performed laboratory and cytokine tests. A.H. and Z.H. acquired the fundings. Z.H. 719 supervised the project.

720 Competing interests: Authors declare no competing interests. 
Fig. 1 | Study design and metabolic profiling in serum samples from mild and severe

724 a, Overview of cohort (including mild $(n=21)$ and severe $(n=23)$ COVID-19 patients and

725 healthy controls $(\mathrm{n}=17))$ and the study design. $\mathbf{b}, \mathrm{t}-\mathrm{SNE}$ plot distributed healthy controls $(\mathrm{n}$

$726=17)$, mild patients $(n=14)$ and severe patients $(n=23)$ according to serum metabolites

727 detected from targeted and untargeted metabolomics. c-d, Volcano plots comparing serum

728 metabolites between mild (c) or severe (d) patients with healthy controls. Significantly

729 increased and decreased metabolites are shown in red and blue, respectively, and

730 metabolites with no statistical significance are shown in gray. Top 5 most significantly

731 increased or decreased metabolites in each group are labeled. e, KEGG metabolic pathways

732 in mild and severe patients enriched by significantly changed serum metabolites in $\mathbf{c}$ and

733 d. f, Schematic depicting the key metabolic pathways altered in response to SARS-CoV-2

734 infection. Gray nodes represent metabolites that were not tested. Metabolite alteration are

735 represented by color intensity, and borders are color-coded by statistical significance.

736 Statistical significance was assessed using two-sided Mann-Whitney U test (c-d), and

737 Fisher's exact test (e) followed by Benjamini-Hochberg multiple comparison test.

\section{Fig. 2 | Metabolite-cytokine correlation in serum samples from COVID-19 patients}

739 a, Metabolic pathways enriched by metabolites that are significantly correlated with each 
740 presented cytokine. $T$ statistics and statistical significance of metabolite-cytokine

741 correlations were assessed based on two-sided $t$ tests of regression coefficients followed

742 by Benjamini-Hochberg multiple comparison test. Mean absolute (abs.) $T$ statistics of

743 metabolites in each pathway were calculated and represented by color intensity. Statistical

744 significance of pathway enrichment analysis was assessed by Fisher's exact test and

745 followed by Benjamini-Hochberg multiple comparison test. b-e, Correlation networks of

746 key CRS-related cytokines and metabolites significantly altered in severe patients. Nodes

747 are color-coded by molecule types and metabolic pathways. Edges are color-coded by

748 association directions. Networks were clustered by fast greedy modularity optimization

749 algorithm. f-h, Chord diagrams of significant metabolite-cytokine correlations with

750 metabolites involved in arginine metabolism (f), purine metabolism (g), tryptophan and

$751 \mathrm{NAD}^{+}$metabolism (h), respectively. Chords are color-coded by association directions

752 consistent with b-e.

753 Fig. 3 | Longitudinal trajectories and metabolite-cytokine correlation in mild COVID-

$754 \quad 19$ patients

755 a, Longitudinal trajectory clustering of significantly changed serum metabolites, cytokines

756 in follow-up patients with mild COVID-19. Metabolite and cytokine abundance in healthy

757 controls were used as base line. Black lines represent the average trajectory for each cluster.

758 b, Heatmap comparison of cytokines at distinct time point in follow-up patients. Log2 fold

759 change of mean cytokine abundance in each interval relative to healthy controls is 
represented by color intensity. c, Enriched KEGG metabolic pathways in each cluster. d,

761 Serum metabolite trajectories based on normalized data in follow-up patients. Blue solid

762 lines pass through the mean of each measurement at the specific time interval, and dotted

763 lines represent the mean of measurements in healthy controls. Generalized addictive model

764 (GAM) regression lines were represented by the black solid lines, with $95 \%$ confidence

765 intervals for the regression line donated by gray filled areas. Data are presented as mean \pm

766 SEM. with individual data points shown. e, Chord diagrams of significant associations

767 between metabolites and core CRS-related cytokines in cluster 1 (left), cluster 2 (middle),

768 cluster 3 and 4 (right), respectively. Statistical significance of was assessed by Fisher's

769 exact test (c) and linear regression model adjusted for age and gender (e) followed by

770 Benjiamini-Hochberg multiple comparison test.

771 Fig. 4 | Targeting metabolism modulates cytokine release in PBMCs ex vivo model

772 a, Schematic representation of the experimental workflow. PBMCs, isolated from 773 peripheral blood of mock-infected or SARS-CoV-2-infected rhesus macaques, were seeded 774 in 96-well plates with vehicle or different drugs dissolved in the medium. 24 hours post775 seeding, cytokine abundance in cell culture was quantified. b-d, Metabolism diagrams and 776 level of indicated cytokines and chemokines measured $24 \mathrm{~h}$ after supplementation of 1.25 $777 \mathrm{mM}$ arginine (b), $0.1 \mathrm{mM}$ IDO1 inhibitor Epacadostat (c), and $0.1 \mathrm{mM}$ IMPDH inhibitor 778 Mycophenolic acid (MPA, d) in PBMCs. Data are presented as mean \pm SEM. with 779 individual data points shown. Statistical significance was assessed using one-way ANOVA 


\section{Extended Data Fig. 1 | Clinical information of mild and severe COVID-19 patients}

782 Clinical information of healthy controls, mild and severe COVID-19 patients. Data are

783 presented as mean \pm SEM. with individual data points shown. Statistical significance was 784 assessed using either one-way ANOVA followed by Benjamini-Hochberg multiple 785 comparison test or unpaired, two-sided $t$-test.

\section{Extended Data Fig. 2 | Overview of metabolomics data in COVID-19 patients}

787 a, General workflow of metabolomic profiling experiments and data analysis. b-c, Partial 788 Least Squares Discriminant Analysis (PLS-DA) of targeted metabolomics data (b) and 789 untargeted metabolomics data (c) of healthy controls, mild and severe COVID-19 patients. 790 d, Venn diagram depicting the number of significantly altered serum metabolites in each 791 group after integrating targeted and untargeted metabolomics data. e, Metabolic pathways 792 enriched based on metabolites progressively increased or decreased in mild and severe 793 patients compared with healthy controls. Statistical significance was assessed by Fisher's 794 exact test and followed by Benjamini-Hochberg multiple comparison test. f-i, Line charts 795 of metabolites which significantly altered in severe patients involved in arginine 796 metabolism (f), TCA cycle (g), tryptophan metabolism (h), NAD+ metabolism (i).

\section{Extended Data Fig. 3 | Overview of cytokine data in COVID-19 patients}


798 a, Alterations in serum cytokine abundance in mild and severe patients compared with 799 healthy controls. Log2 fold change is represented by color intensity. Shape represents 800 alteration significance, and FDR is size-coded. b, Violin plots comparing serum cytokines 801 abundance in healthy controls, mild patients and severe patients. Data are presented as 802 mean and quantiles with individual data points shown. Statistical significance was assessed 803 using two-sided Mann-Whitney $U$ test followed by Benjamini-Hochberg multiple 804 comparison test. 19 patients

807 a, Schematic diagram of sample collection for follow-up patients. Blue squares indicate 808 the time of serum collection; red circles represent the time of symptoms onset; dark blue 809 lines represent the time of hospitalization. b-c, Longitudinal antibodies (b), $\log 10$ 810 transformed cytokine and chemokine concentration (c) trajectories in an interval of 3 days.

811 Blue solid lines pass through the mean of each measurement at the specific time interval, 812 and dotted lines represent the mean of measurements in healthy controls. GAM regression 813 lines are indicated by the black solid lines, with $95 \%$ confidence intervals for the regression 814 lines donated by gray filled areas. Data are presented as mean $\pm \mathrm{SEM}$. with individual data 815 points shown.

816 Extended Data Fig. 5 | Longitudinal metabolite trajectories in follow-up mild COVID- 
818 a, Heatmap comparison of metabolites at distinct time point in follow-up patients in each

819 cluster. Log2 fold change of metabolite abundance in each interval relative to healthy

820 controls is represented by color intensity. b, Serum metabolite trajectories based on

821 normalized data in follow-up patients. Blue solid lines pass through the mean of each

822 measurement at the specific time interval, and dotted lines represent the mean of

823 measurements in healthy controls. GAM regression lines are represented by the black solid

824 lines, with 95\% confidence intervals for the regression donated by gray filled areas. Data

825 are presented as mean \pm SEM. with individual data points shown.

Extended Data Fig. 6 |Cytokine abundance after supplementation with arginine

827 Levels of indicated cytokines and chemokines measured $24 \mathrm{~h}$ after supplementation of 1.25

$828 \mathrm{mM}$ arginine in isolated PBMCs derived from mock-infected or SARS-CoV-2-infected

829 rhesus macaques. Data are presented as mean \pm SEM. with individual data points shown.

830 Statistical significance was assessed using one-way ANOVA followed by Benjamini-

831 Hochberg multiple comparison test.

a, Ratio of kynurenine (Kyn) to tryptophan (Try) in healthy controls and COVID-19

834 patients. b, Levels of indicated cytokines and chemokines measured $24 \mathrm{~h}$ after $0.1 \mathrm{mM}$ 
IDO1 inhibitor Epacadostat treatment in isolated PBMCs derived from mock-infected or

SARS-CoV-2-infected rhesus macaques. Data are presented as mean \pm SEM with individual data points shown. Statistical significance was assessed using one-way ANOVA followed by Benjamini-Hochberg multiple comparison test.

\section{Extended Data Fig. 8 |Cytokine abundance after treatment with IMPDH inhibitor}

Levels of indicated cytokines and chemokines measured $24 \mathrm{~h}$ after treatment of $0.1 \mathrm{mM}$ IMPDH inhibitor mycophenolic acid (MPA) in isolated PBMCs derived from mockinfected or SARS-CoV-2-infected rhesus macaques. Data are presented as mean $\pm \mathrm{SEM}$. with individual data points shown. Statistical significance was assessed using one-way ANOVA followed by Benjamini-Hochberg multiple comparison test.

\section{Extended Data Fig. 9 | Viral RNA quantification in isolated PBMCs ex vivo}

a, PBMCs were isolated from uninfected rhesus macaque and were either mock or SARS-

CoV-2 infected and cultured for $24 \mathrm{~h}$ under different treatment conditions. Viral RNA in PBMCs were then assessed by quantitative PCR analysis. b, Levels of SARS-CoV-2 RNA in PBMCs quantified $24 \mathrm{~h}$ after infected and supplementation of $1.25 \mathrm{mM}$ arginine, 0.1 mM IDO1 inhibitor Epacadostat, and 0.1mM IMPDH inhibitor Mycophenolic acid (MPA), respectively. Statistical significance was assessed using one-way ANOVA followed by Benjamini-Hochberg multiple comparison test. Data are presented as mean \pm SEM. with individual data points shown. 
854 Supplementary Tables

855 Extended Data Table 1 | Clinical characteristics of healthy controls and COVID-19

856 patients

857 Extended Data Table 2 | Normalized targeted and untargeted metabolomics data 


\section{Figure 1}

a

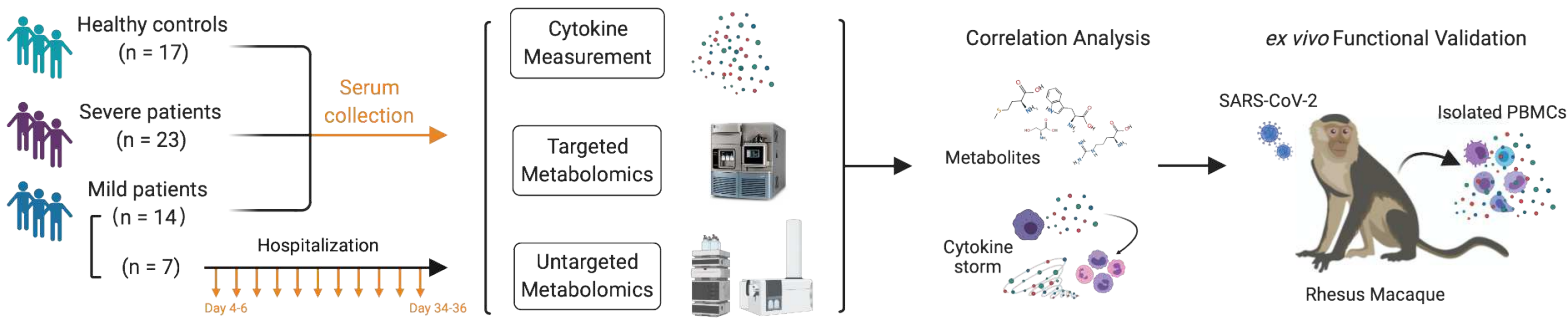

b

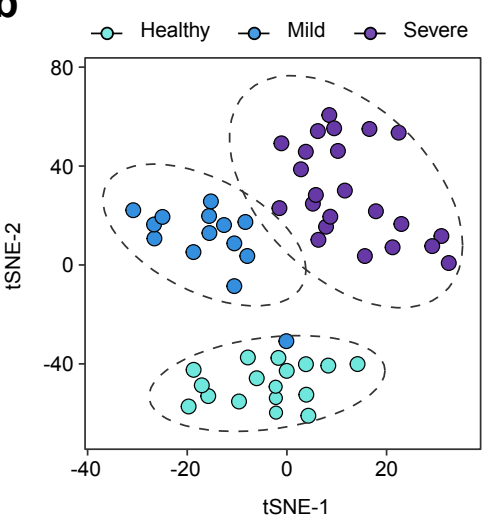

e

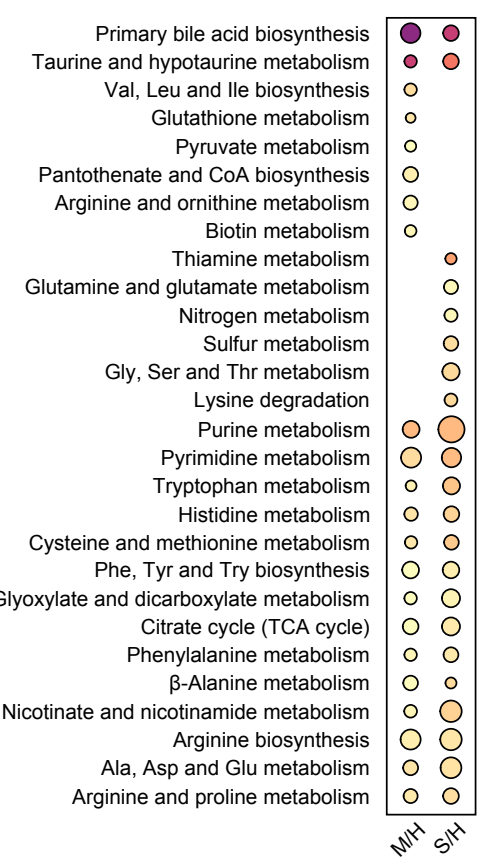

abs. $\log _{2}$ (Fold change) $\quad-\log _{10}(P$ value $)$

$\begin{array}{llllll} & & \vdots & & \bigcirc & \bigcirc \\ 0.5 & 1.5 & 2.5 & 2 & 6 & 10\end{array}$
C

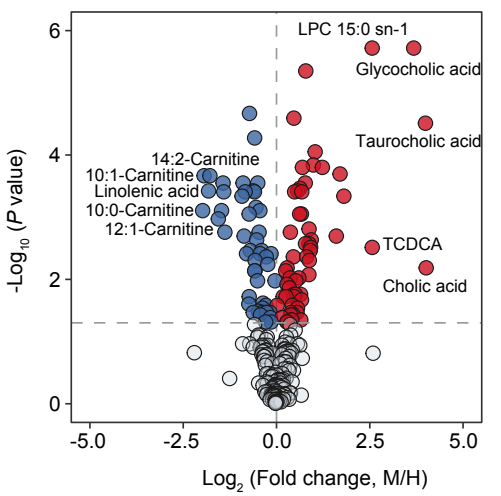

d

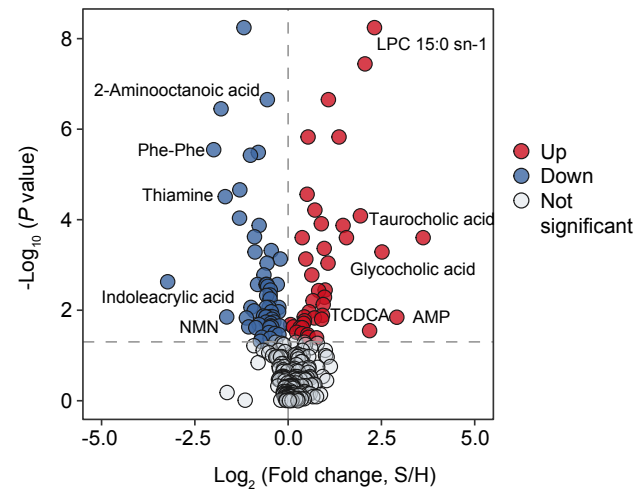

f
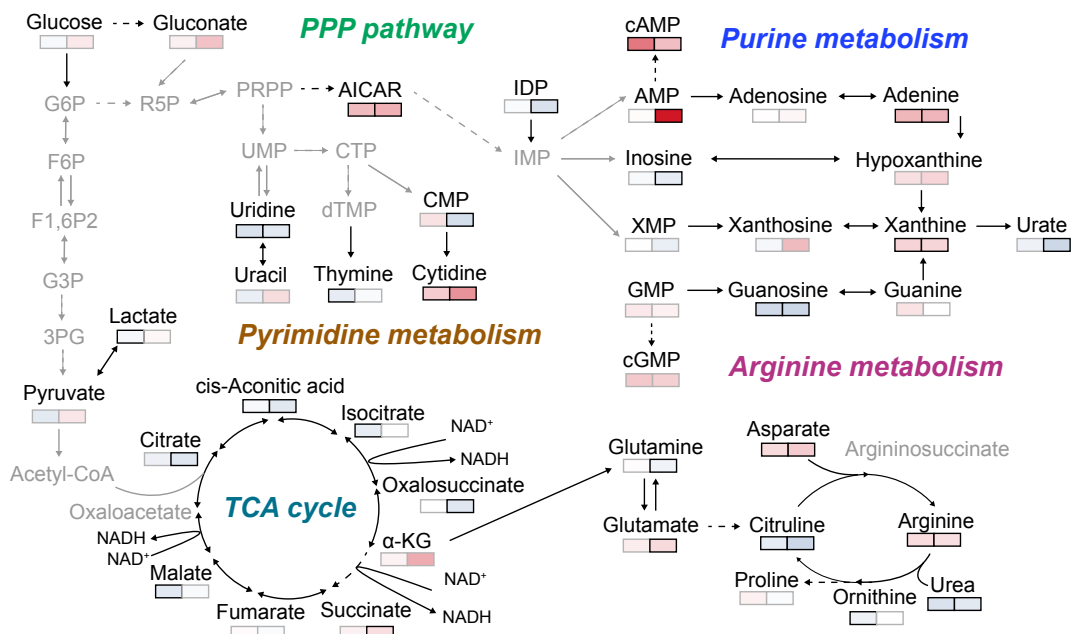

Tryptophan metabolism

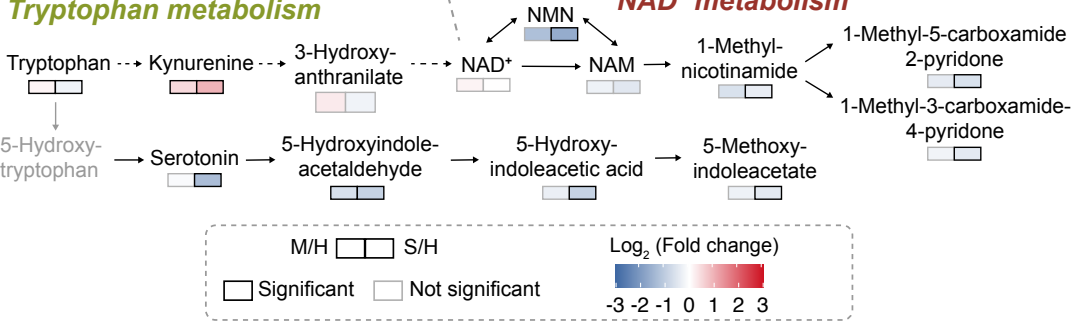




\section{Figure 2}

a

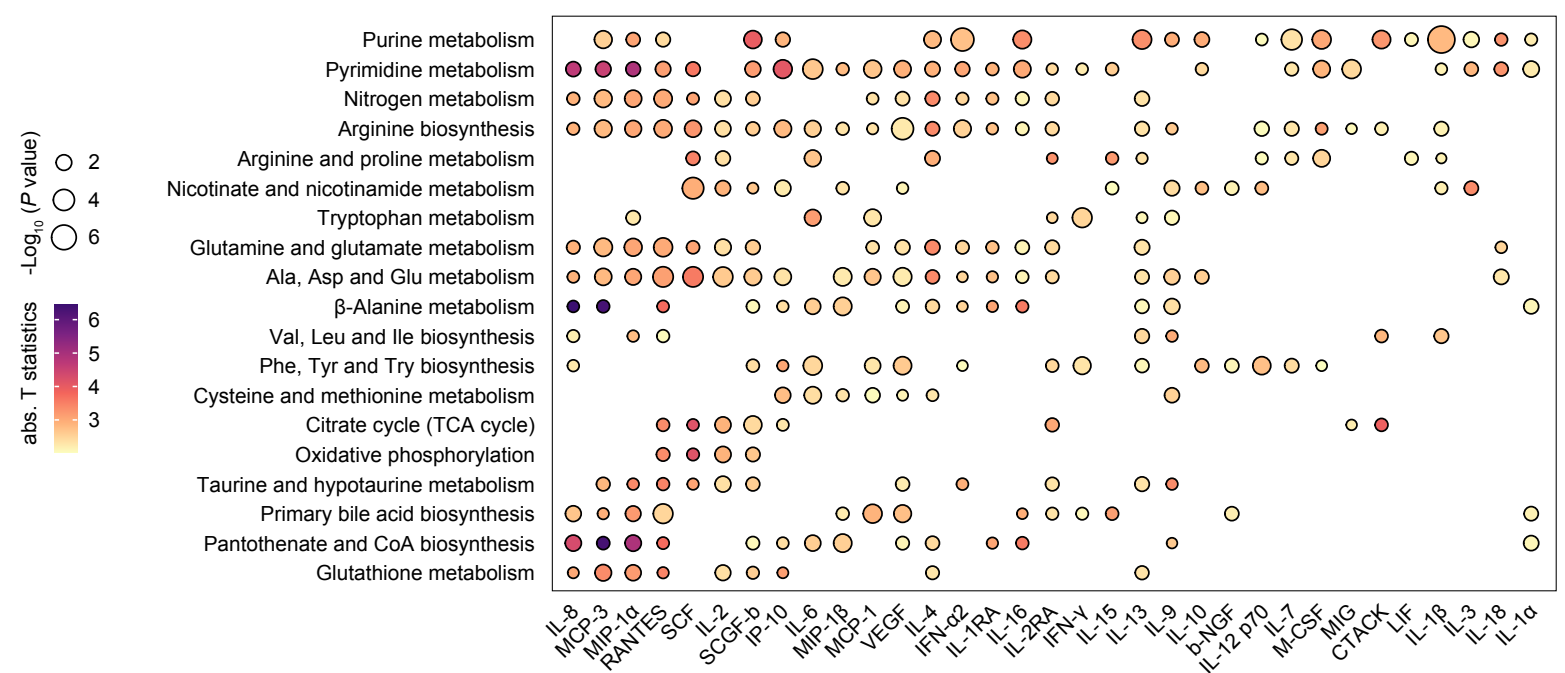

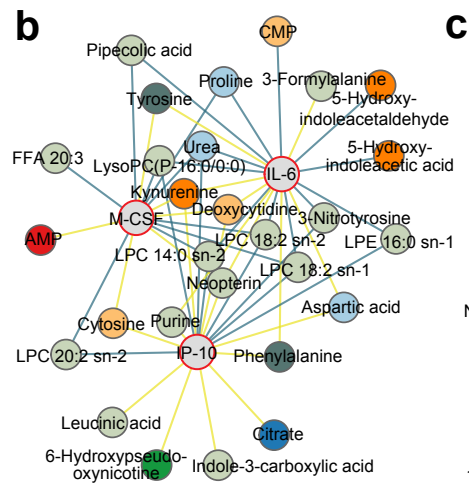

C a-ketoisovaleric @iid Trimethyllysine

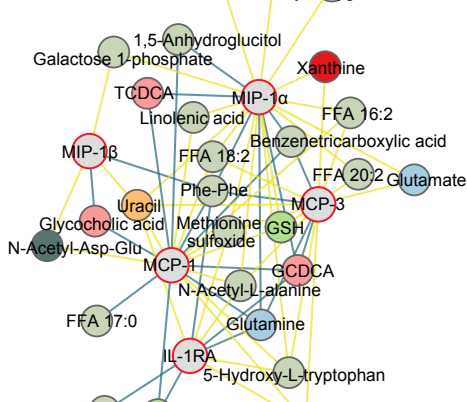

18:0-Carntine L-Honocysteine 5 -H (droxylysine

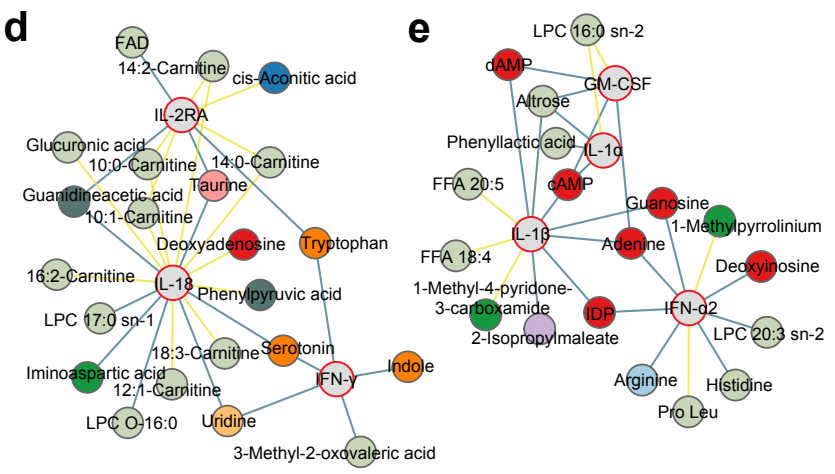

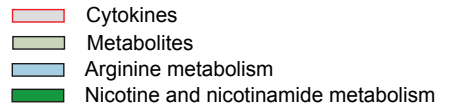

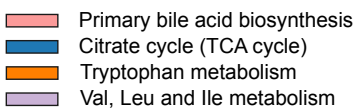

f
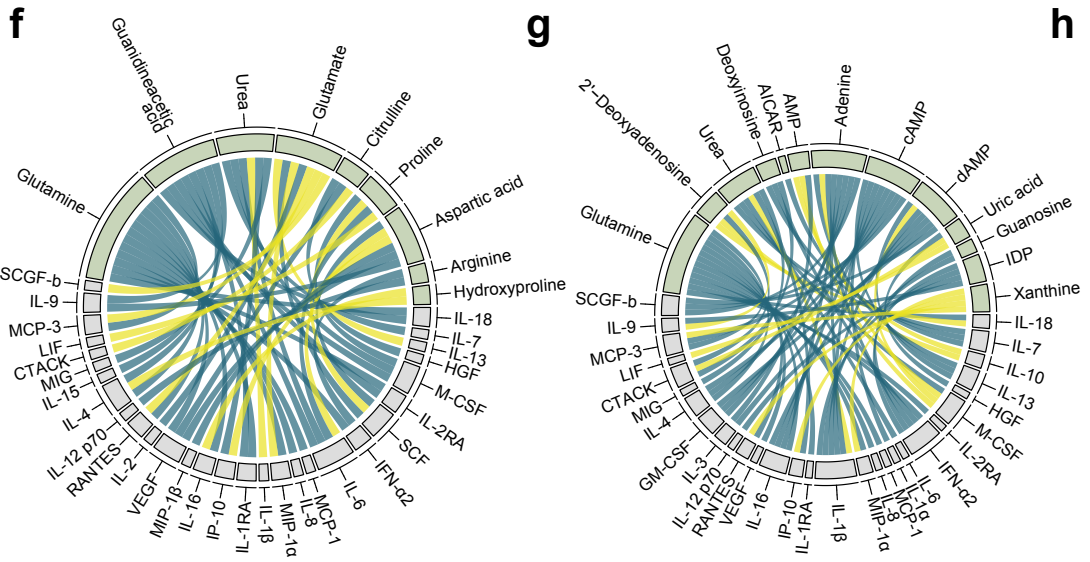

Purine metabolism

Prymidine metabolism ___ Positive correlation

Cysteine and methionine metabolism _ _ Negative correlation h

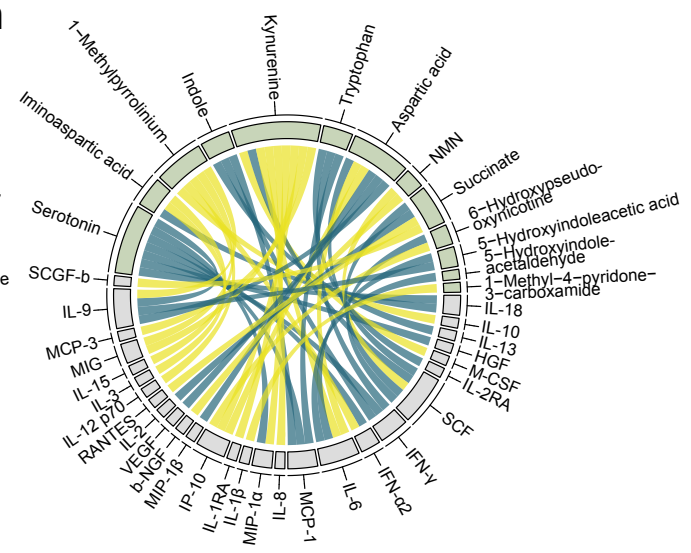


a

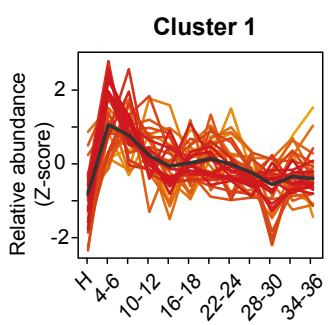

Days after symptoms onset

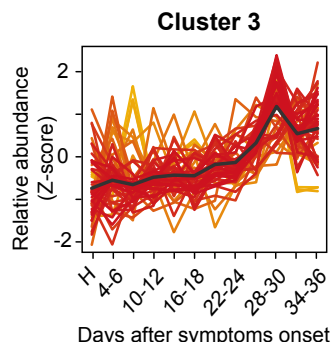

C

Primary bile acid biosynthesis Phenylalanine metabolism Ala, Asp and Glu metabolism Pyrimidine metabolism Purine metabolism
Taurine and hypotaurine metabolism Nicotinate and nicotinamide metabolism Cysteine and methionine metabolism Citrate cycle (TCA cycle)

Arginine biosynthesis Arginine and proline metabolism
Glyoxylate and dicarboxylate metabolism Gly, Ser and Thr metabolism Glycerophospholipid metabolism Glutathione metabolism

Val, Leu and Ile degradation Val, Leu and Ile biosynthesis Tryptophan metabolism Phe, Tyr and Try biosynthesis

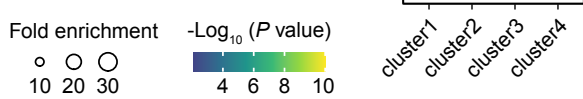

$\begin{array}{ccc}\circ & \bigcirc & \bigcirc \\ 10 & 20 & 30\end{array}$ $\begin{array}{llll}4 & 6 & 8 & 10\end{array}$

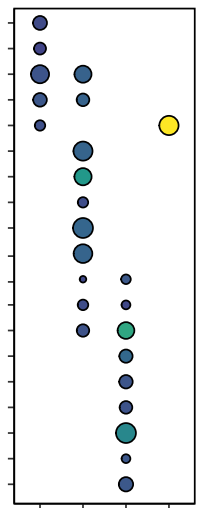

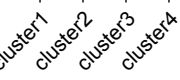

Cluster 2

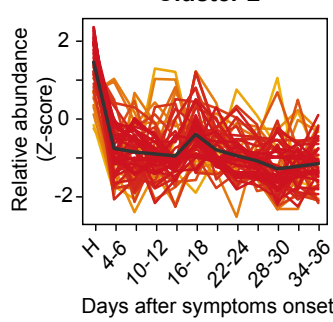

Cluster 4

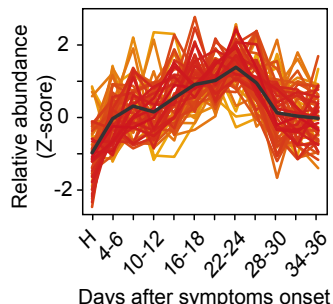

b

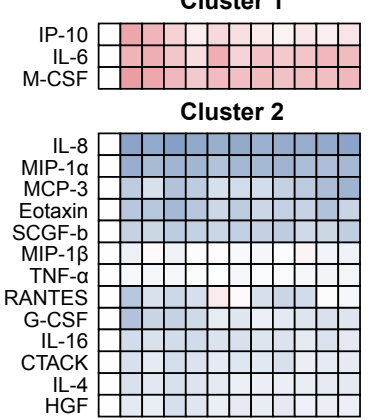

Cluster 3

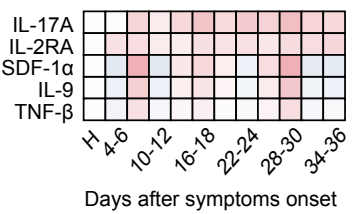

Cluster 4

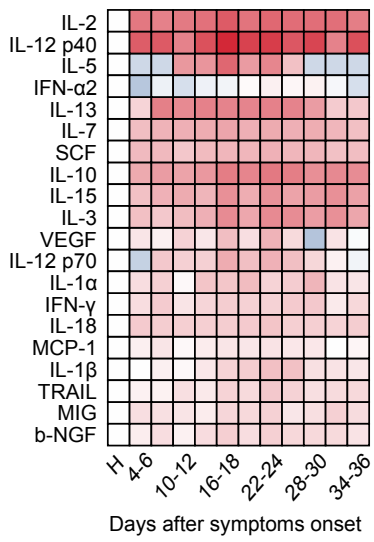

$\log _{2}$ (Fold change) Relative to healthy controls

$\begin{array}{lll}-4 & 0 & 4\end{array}$

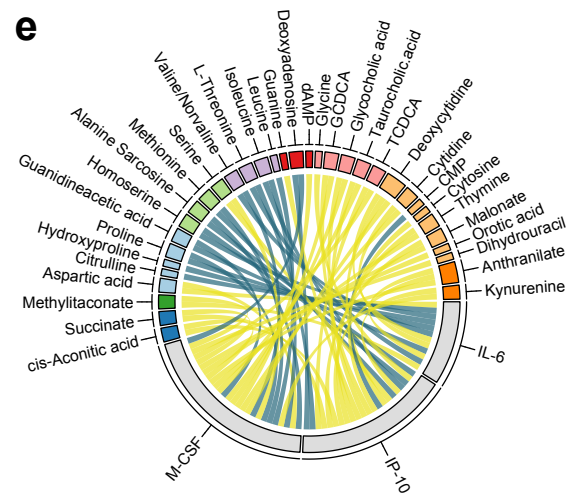

d
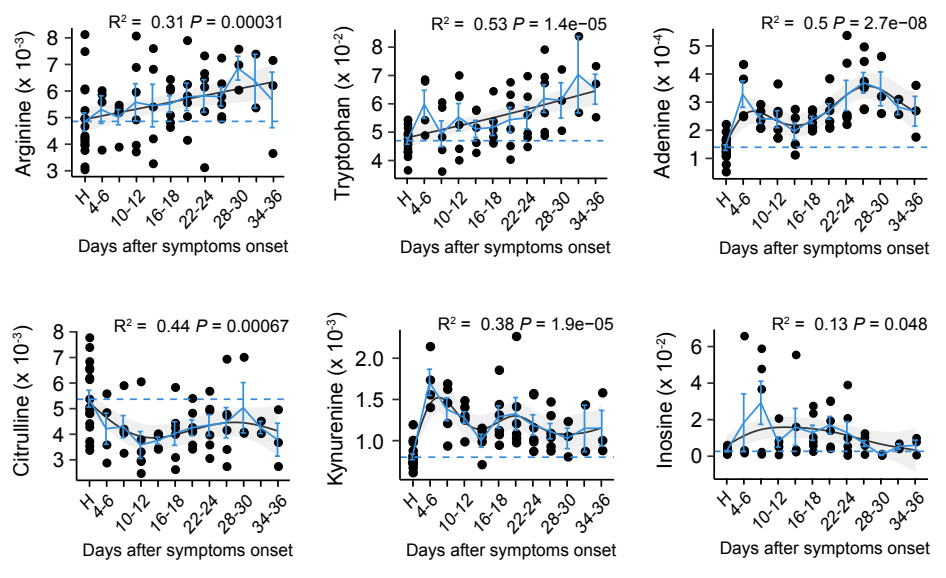
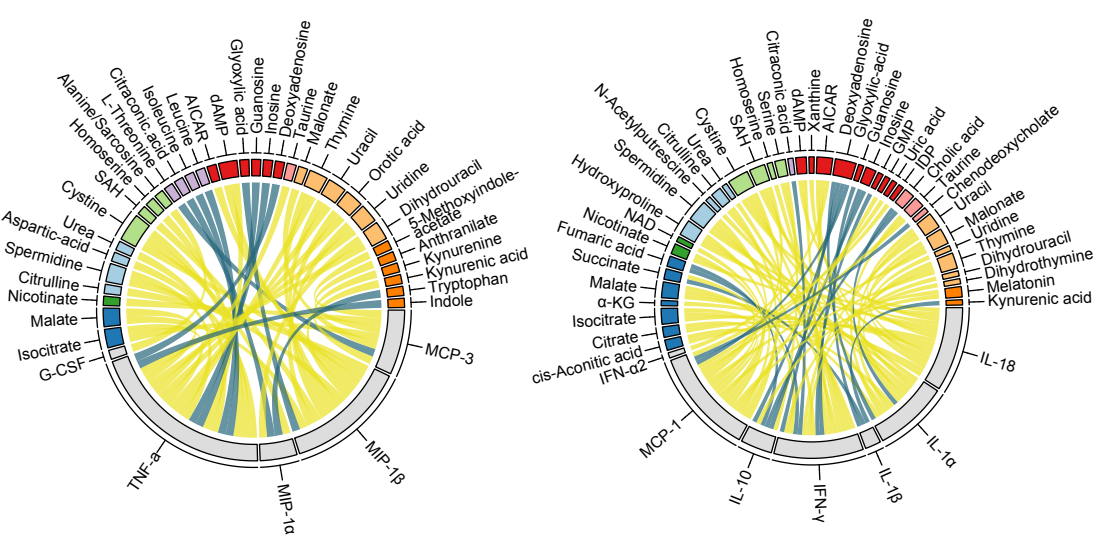

$\square$ Cytokines
$\square$ Arginine metabolism
Nicotine and nicotinamide metabolism
Cysteine and methionine metabolism
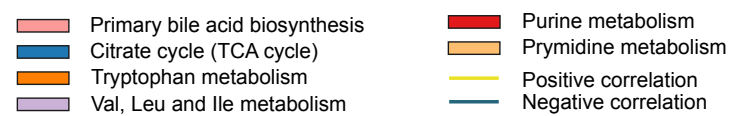

Prymidine metabolism
$\square$ Positive correlation

Tryptophan metabolism

Negative correlation 
Figure 4

a
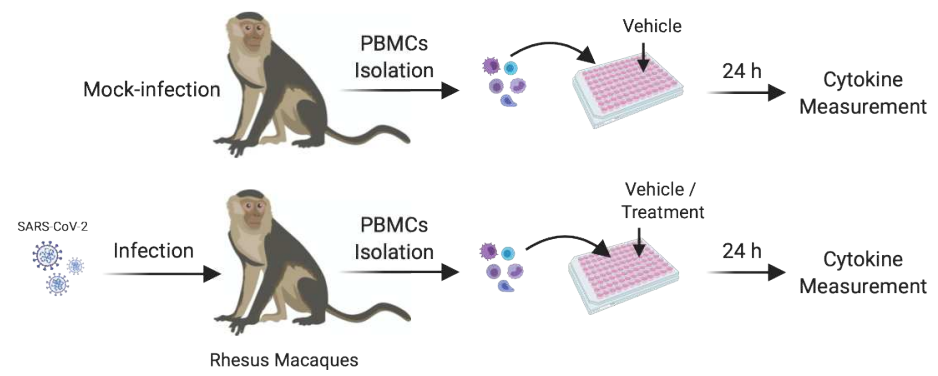

b
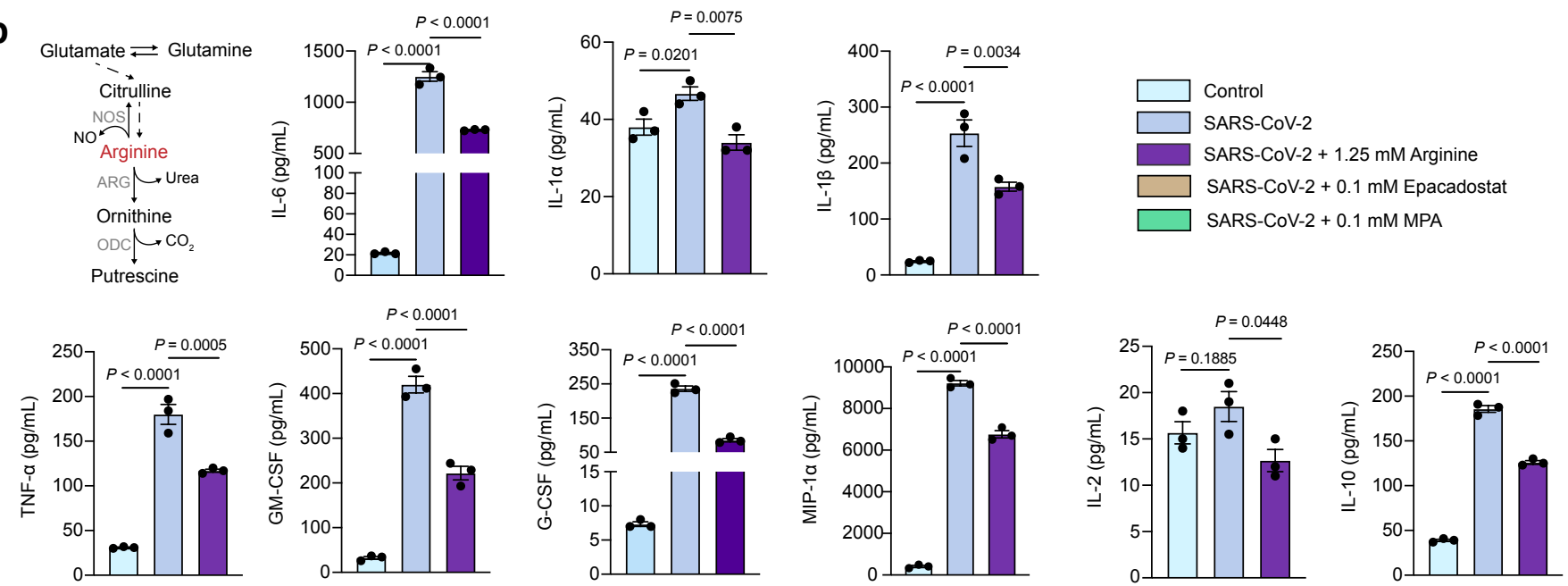

C
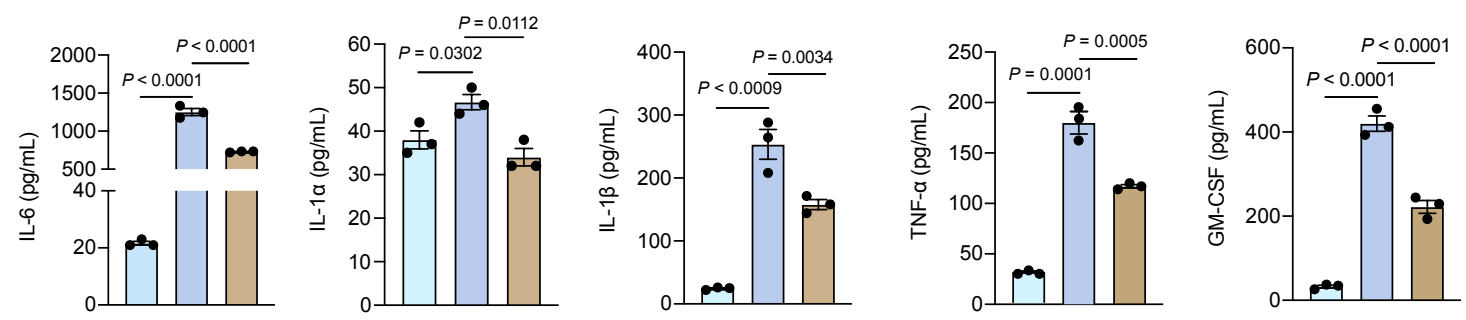

d
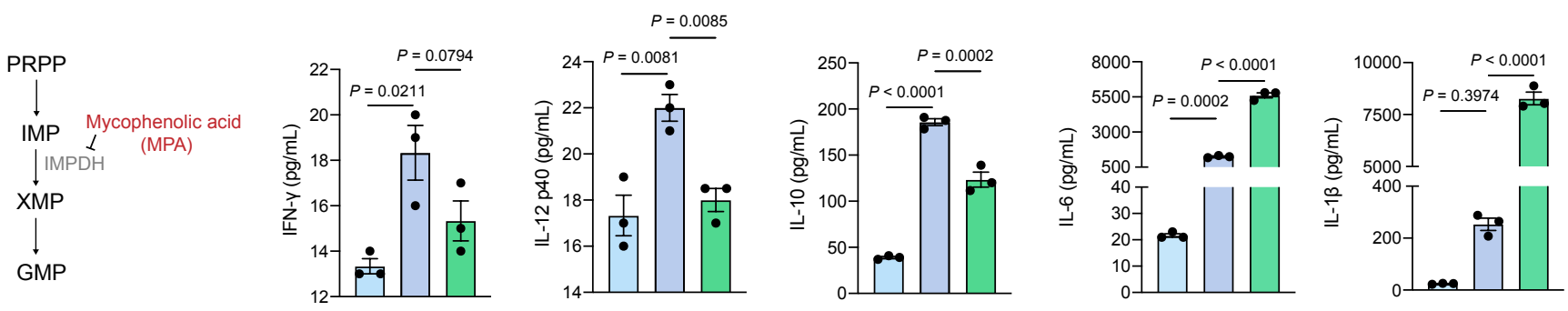


\section{Extended Data Figure 1}
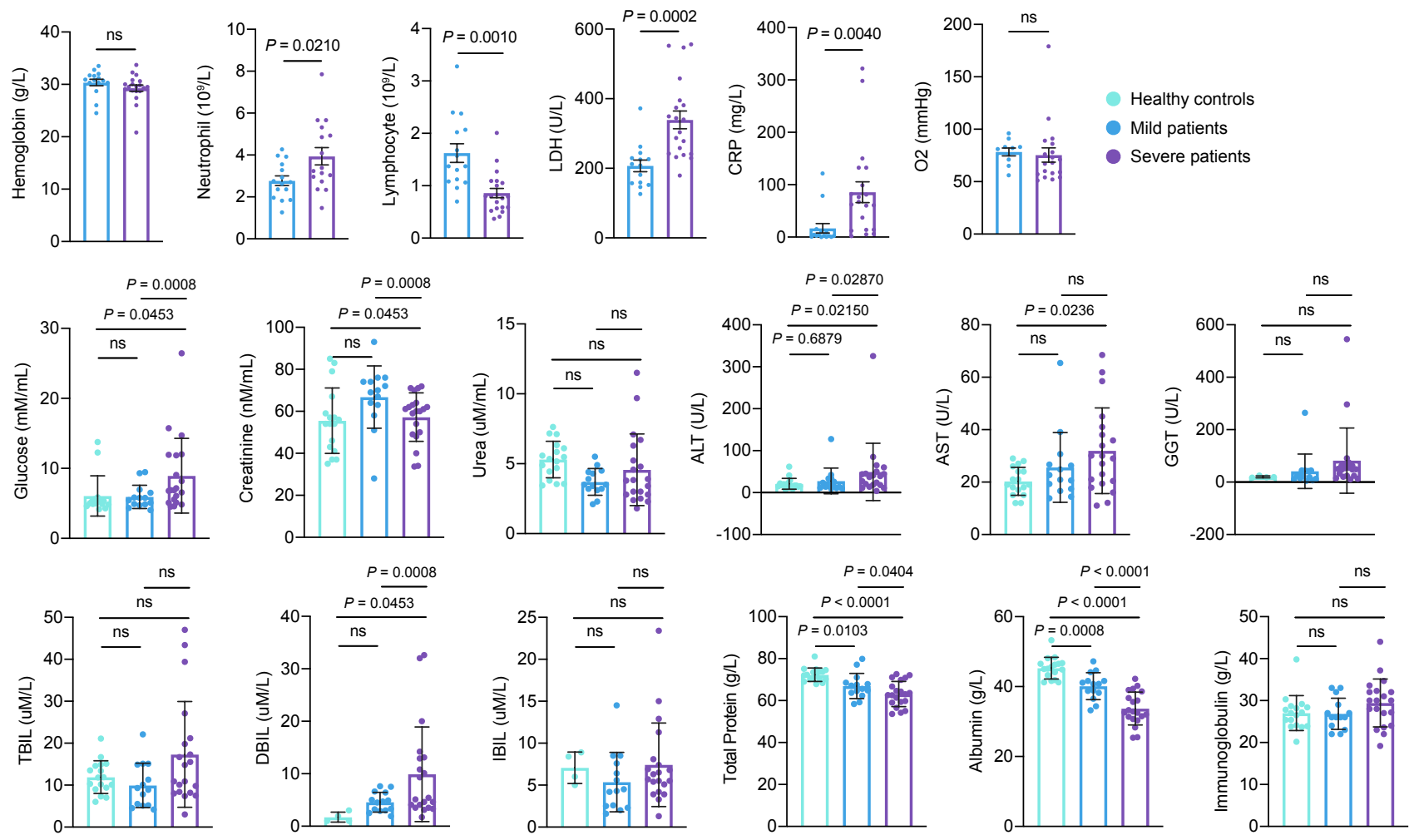


\section{Extended Data Figure 2}

a

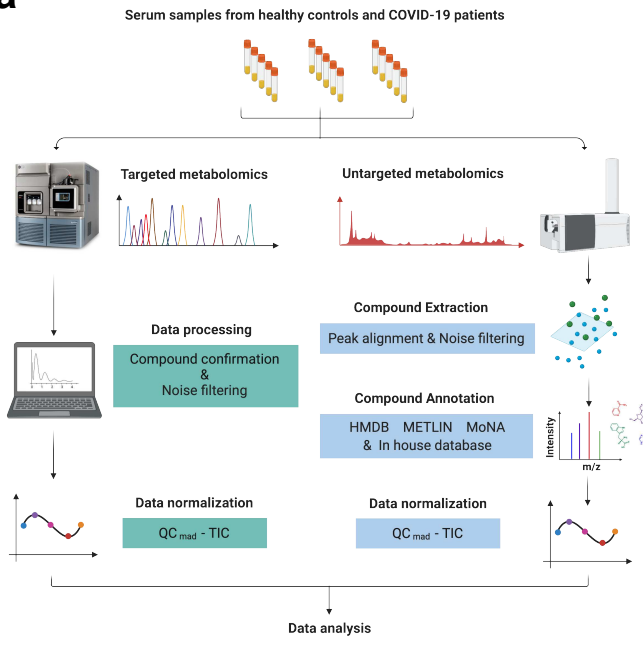

b

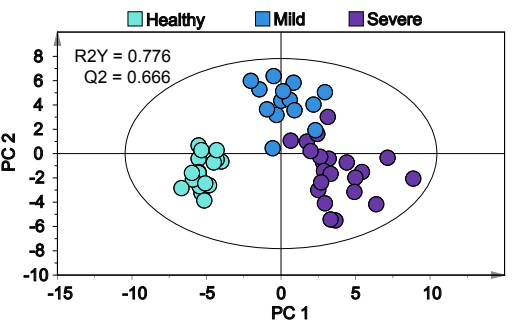

C

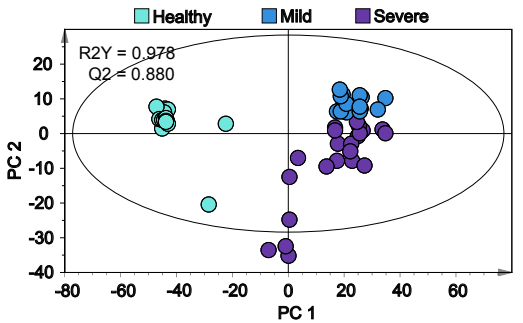

d

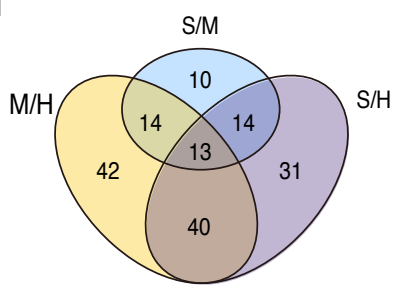

g

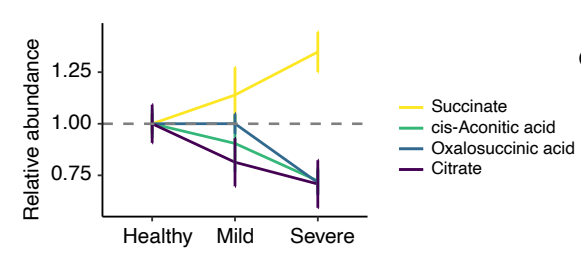

Purine metabolism Ala, Asp and Glu metabolism Nicotinate and nicotinamide metabolism Tryptophan metabolism Histidine metabolism Citrate cycle (TCA cycle) Arginine biosynthesis Phenylalanine metabolism Glyoxylate and dicarboxylate metabolism Pyrimidine metabolism Sulfur metabolism Phe, Tyr and Try biosynthesis Gly, Ser and Thr metabolism Cysteine and methionine metabolism Arginine and proline metabolism Fold enrichment

$\begin{array}{lll}10 & 15 \quad 20\end{array}$

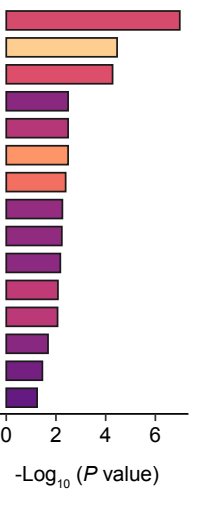

h

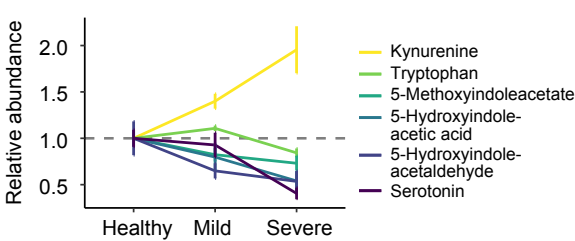

i

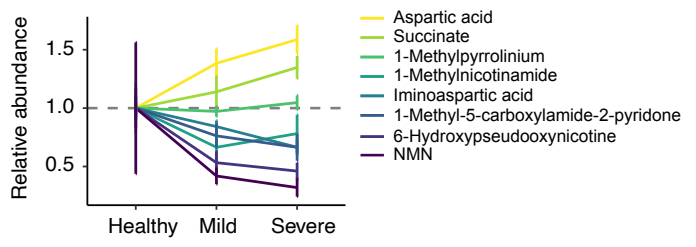




\section{Extended Data Figure 3}

a

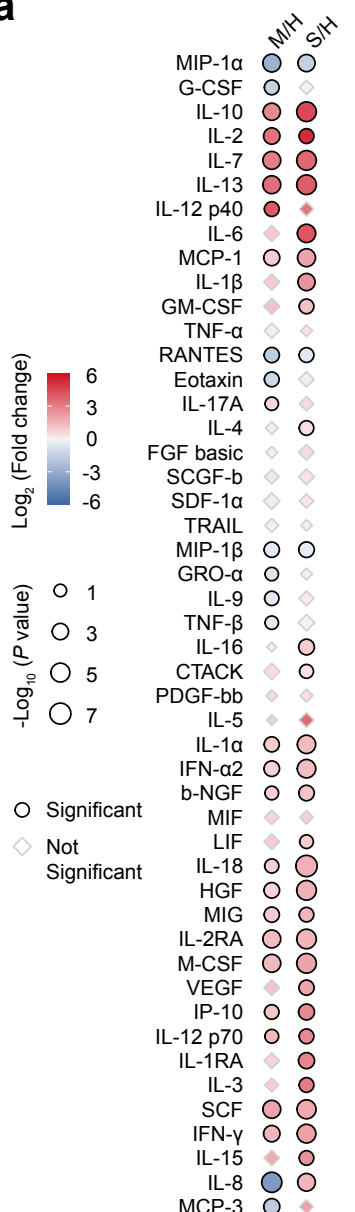

b
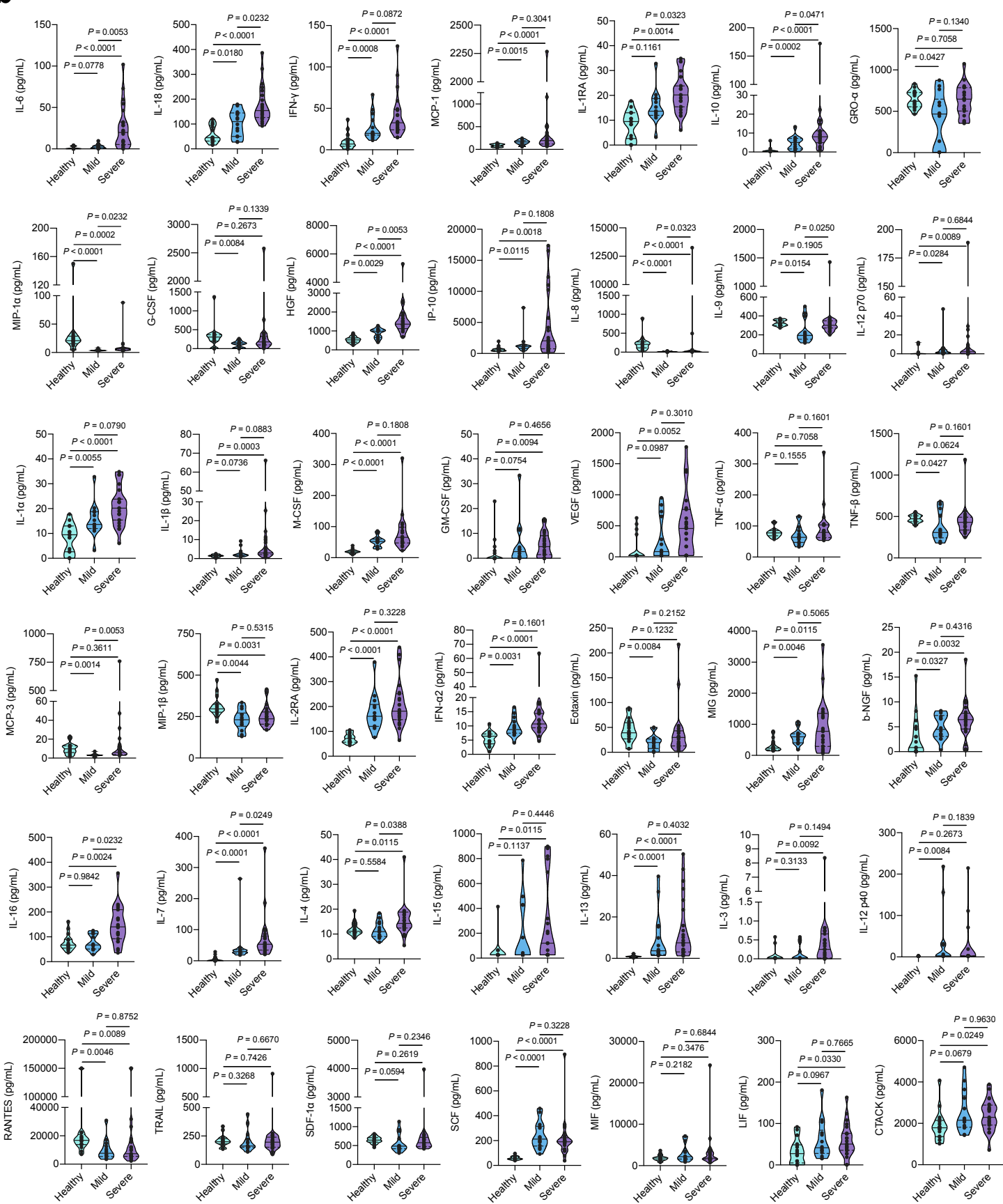

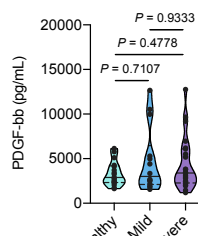
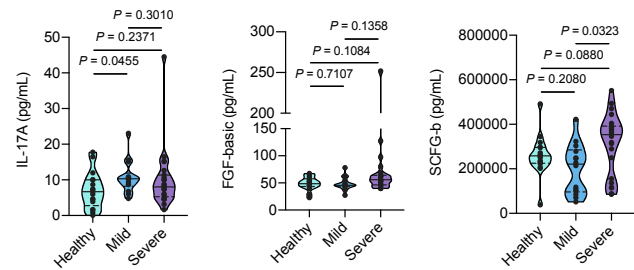


\section{Extended Data Figure 4}

a

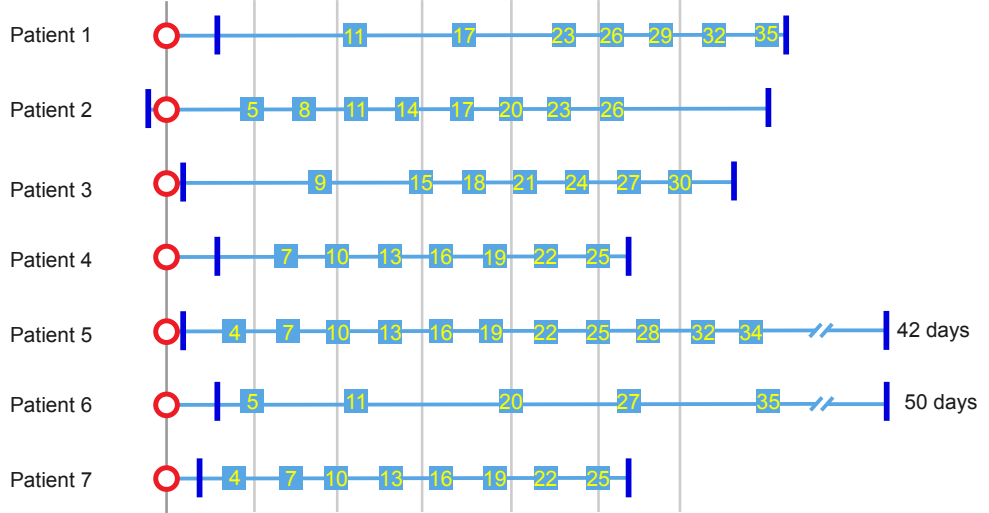

16 years, male

37 years, male

50 years, female

63 years, female

63 years, male

18 years, male

46 years, female

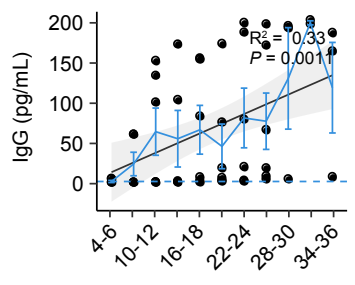

Days after symptoms onset

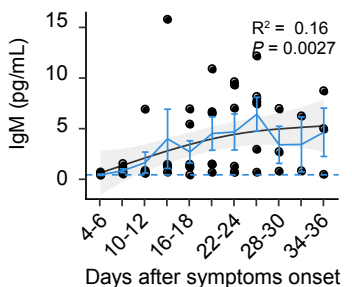

C

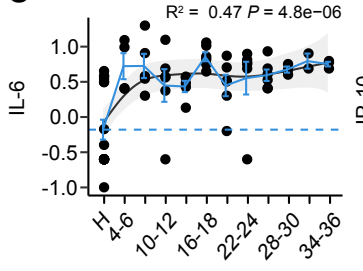

Days after symptoms onset

$R^{2}=0.31 P=0.00044$
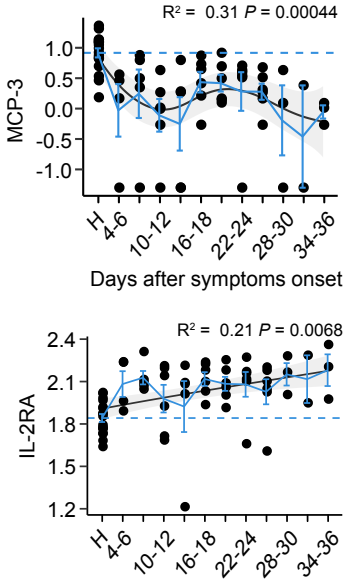

Days after symptoms onset

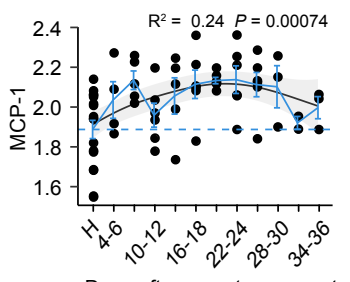

Days after symptoms onset

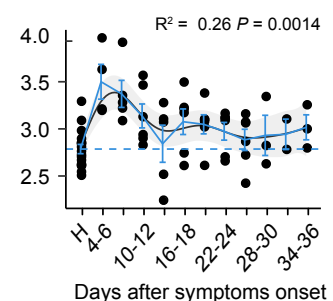

2.0
Days after symptoms onset

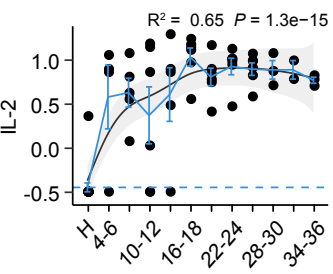

Days after symptoms onset

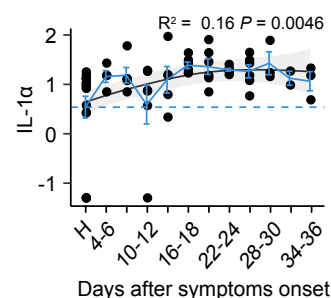

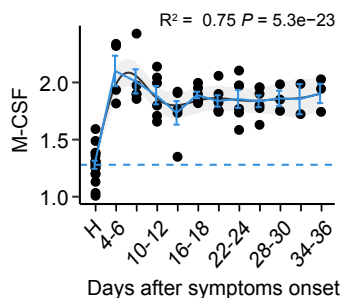

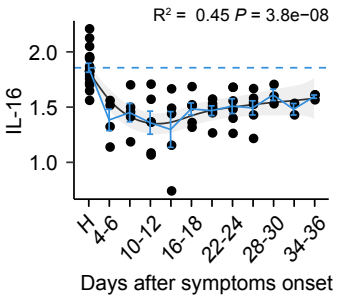

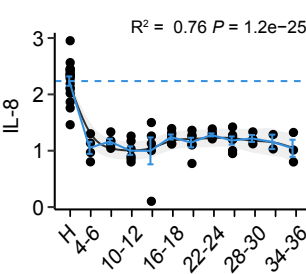

Days after symptoms onset
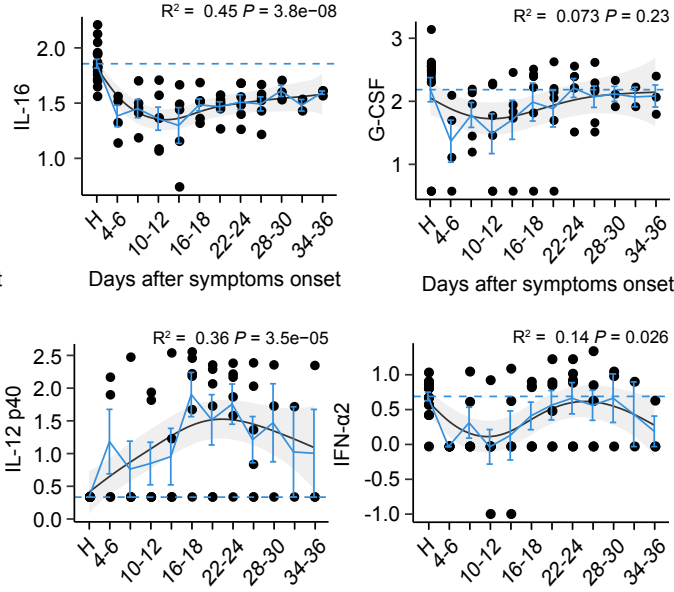

Days after symptoms onset

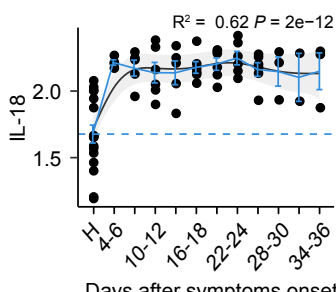

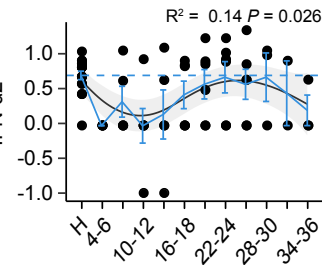

Days after symptoms onset

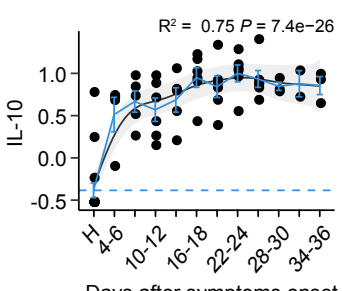

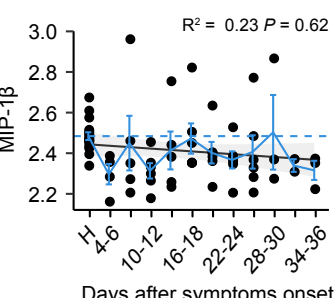

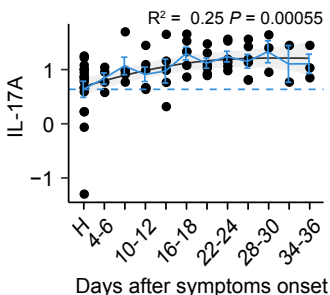

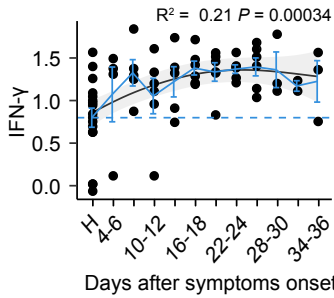

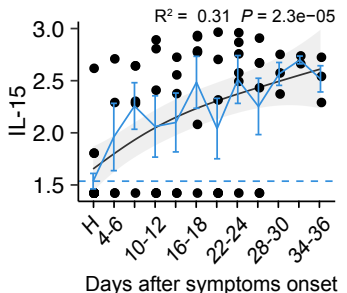




\section{Extended Data Figure 5}

a
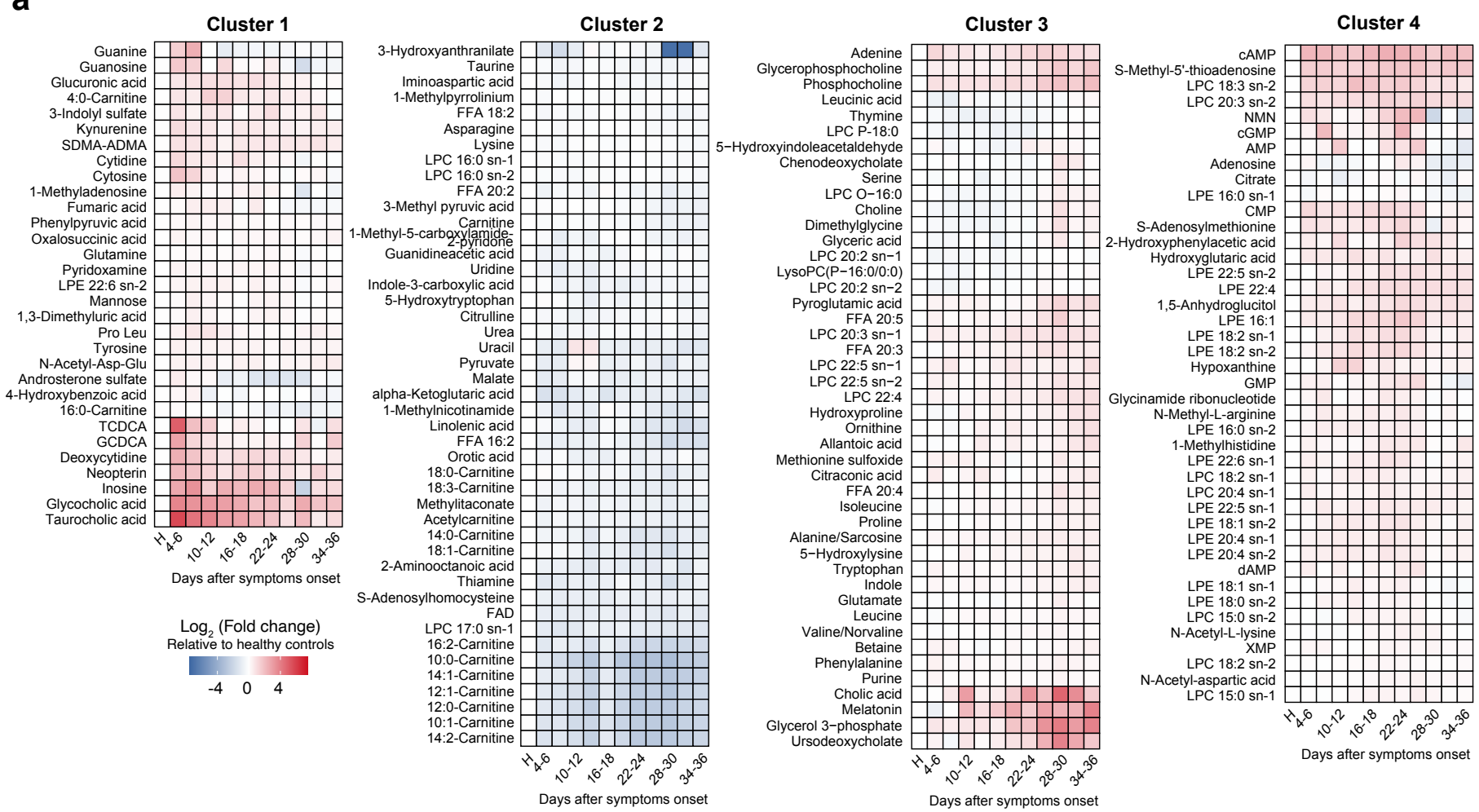

b
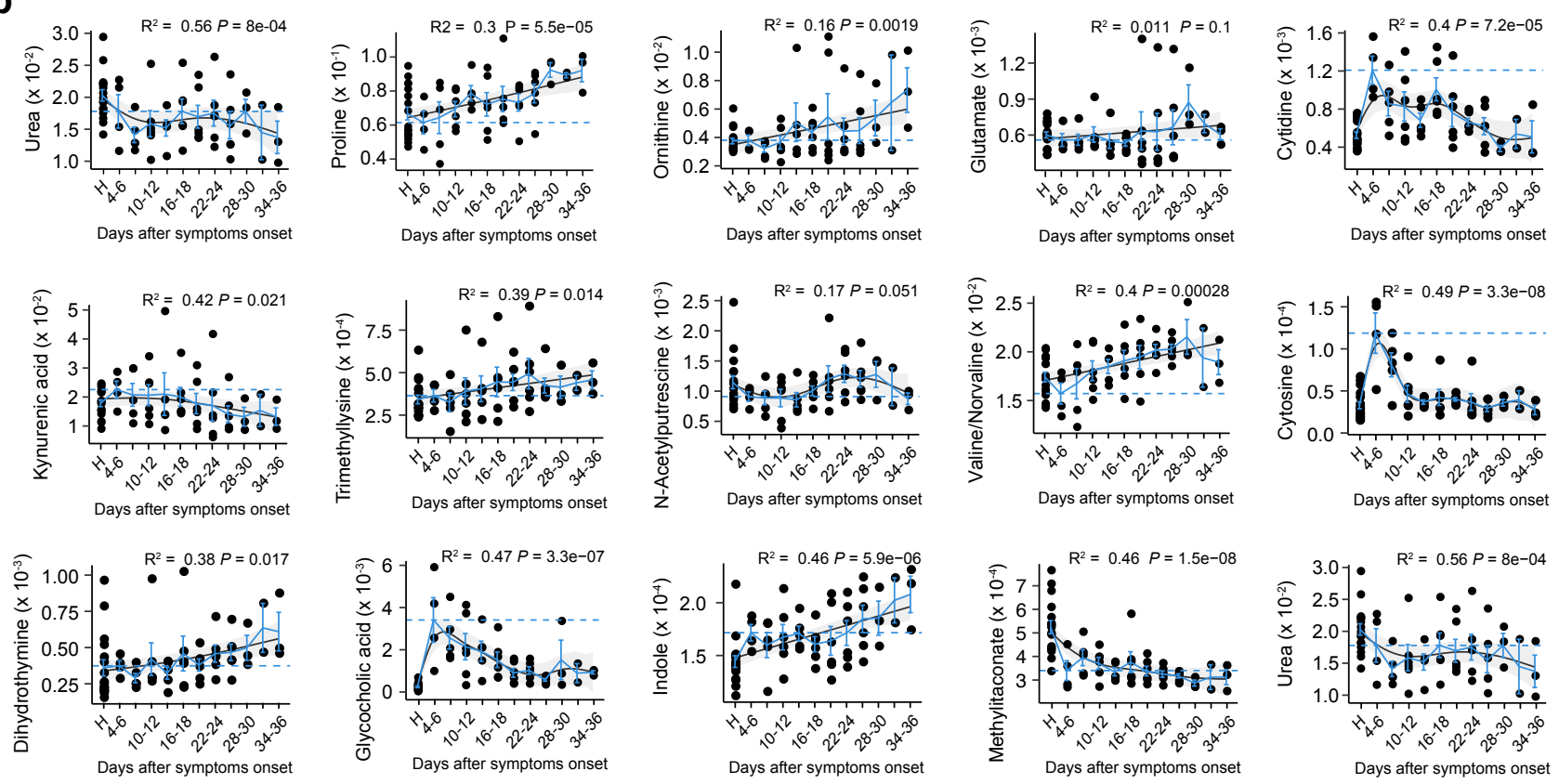


\section{Extended Data Figure 6}
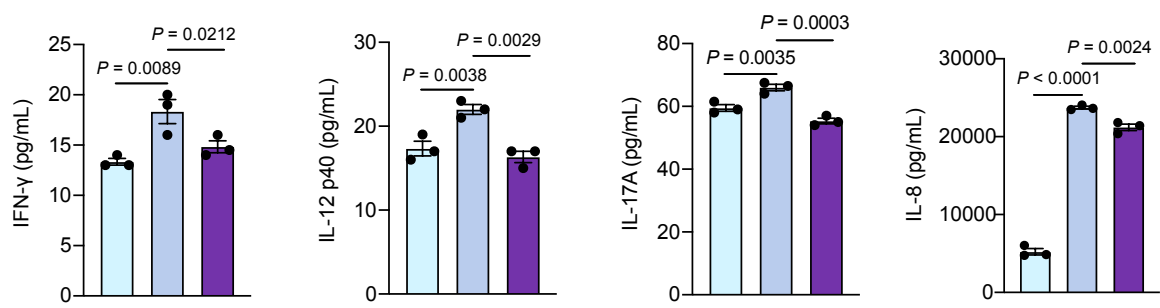

Control

SARS-CoV-2
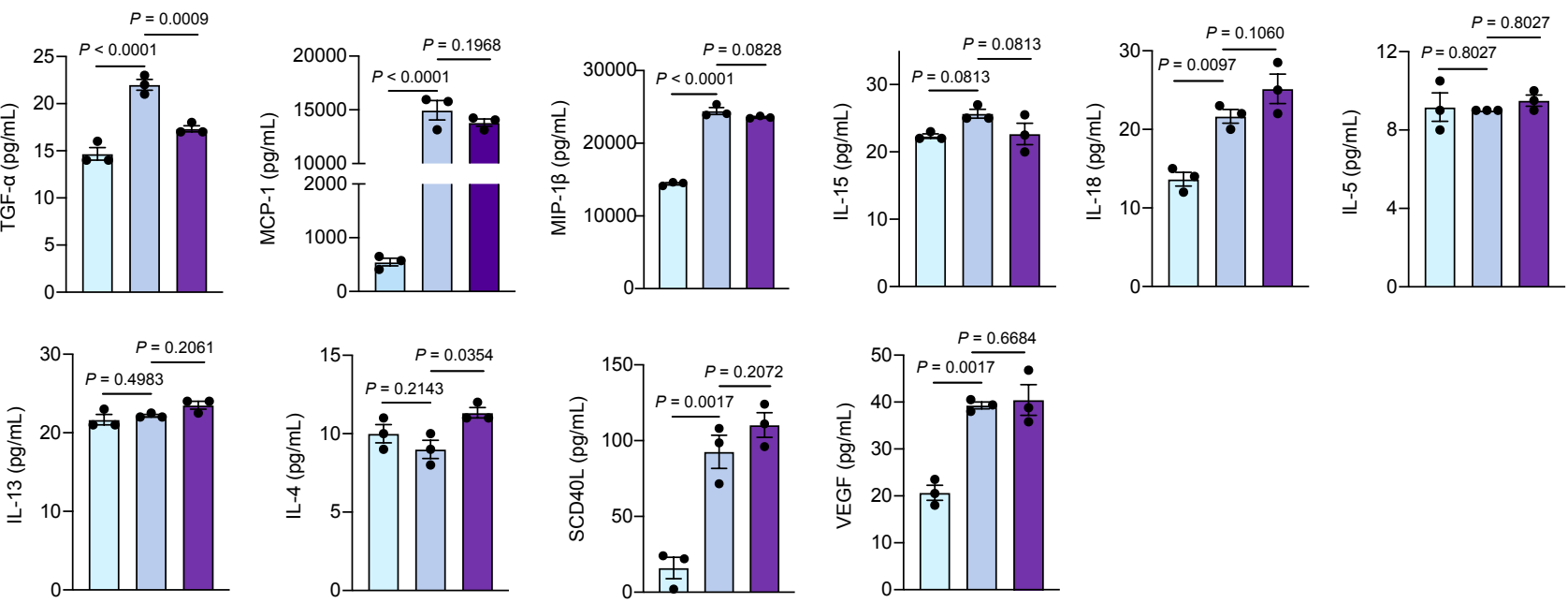


\section{Extended Data Figure 7}

a
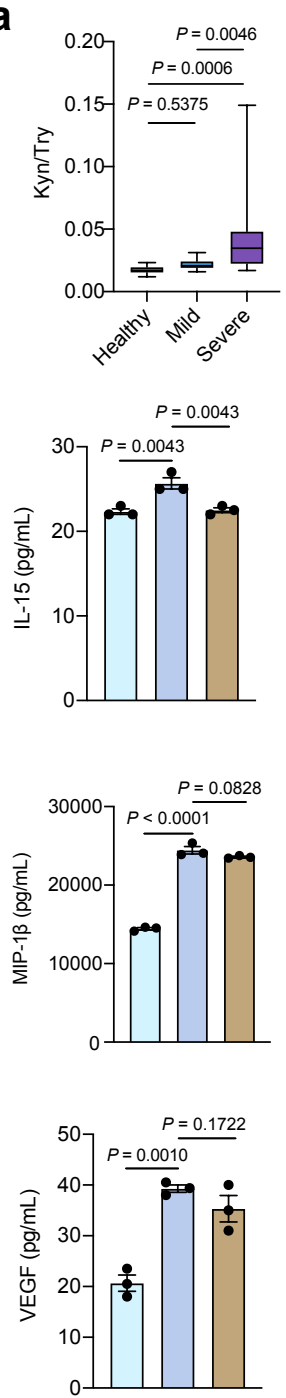

b
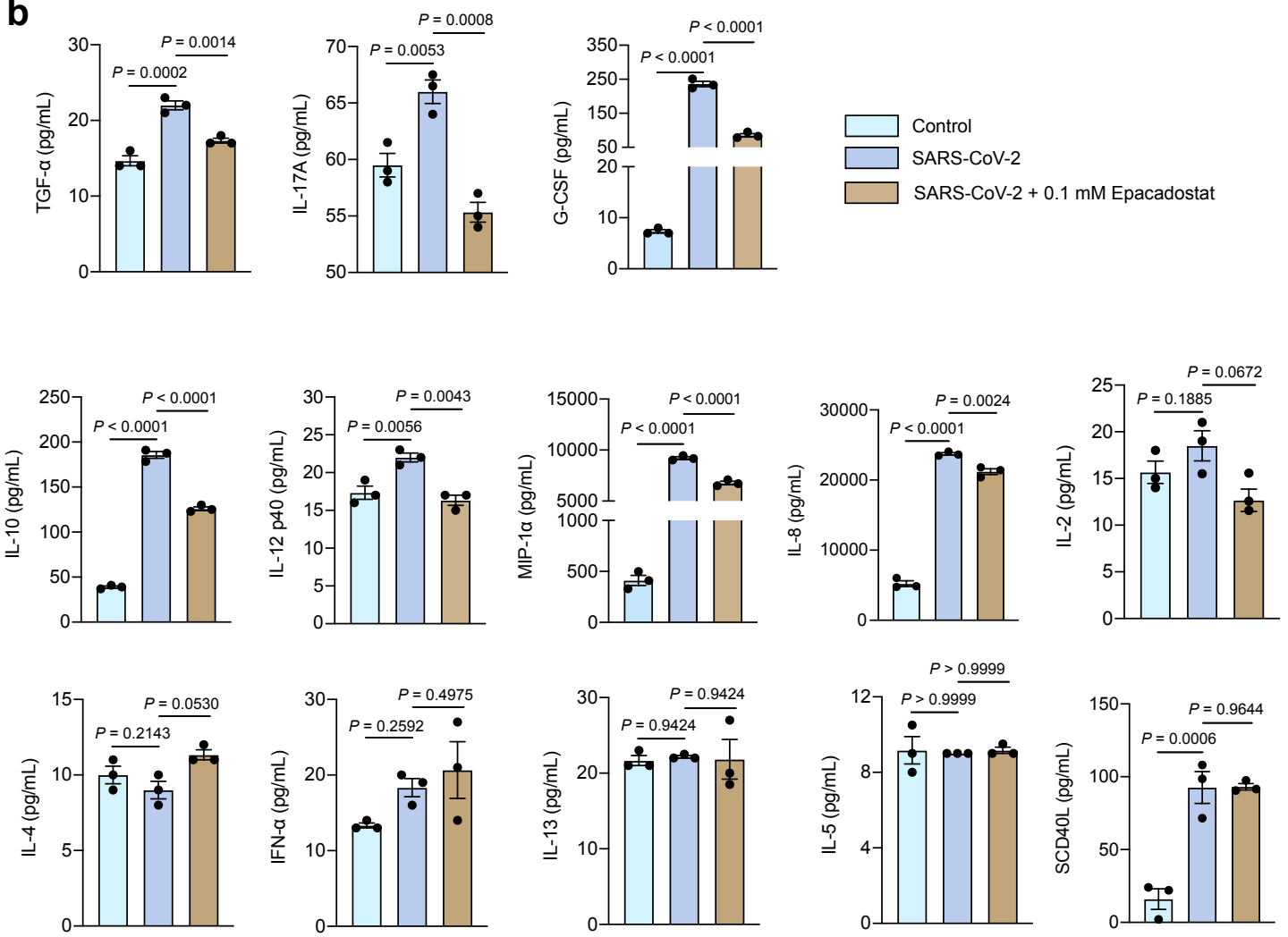


\section{Extended Data Figure 8}
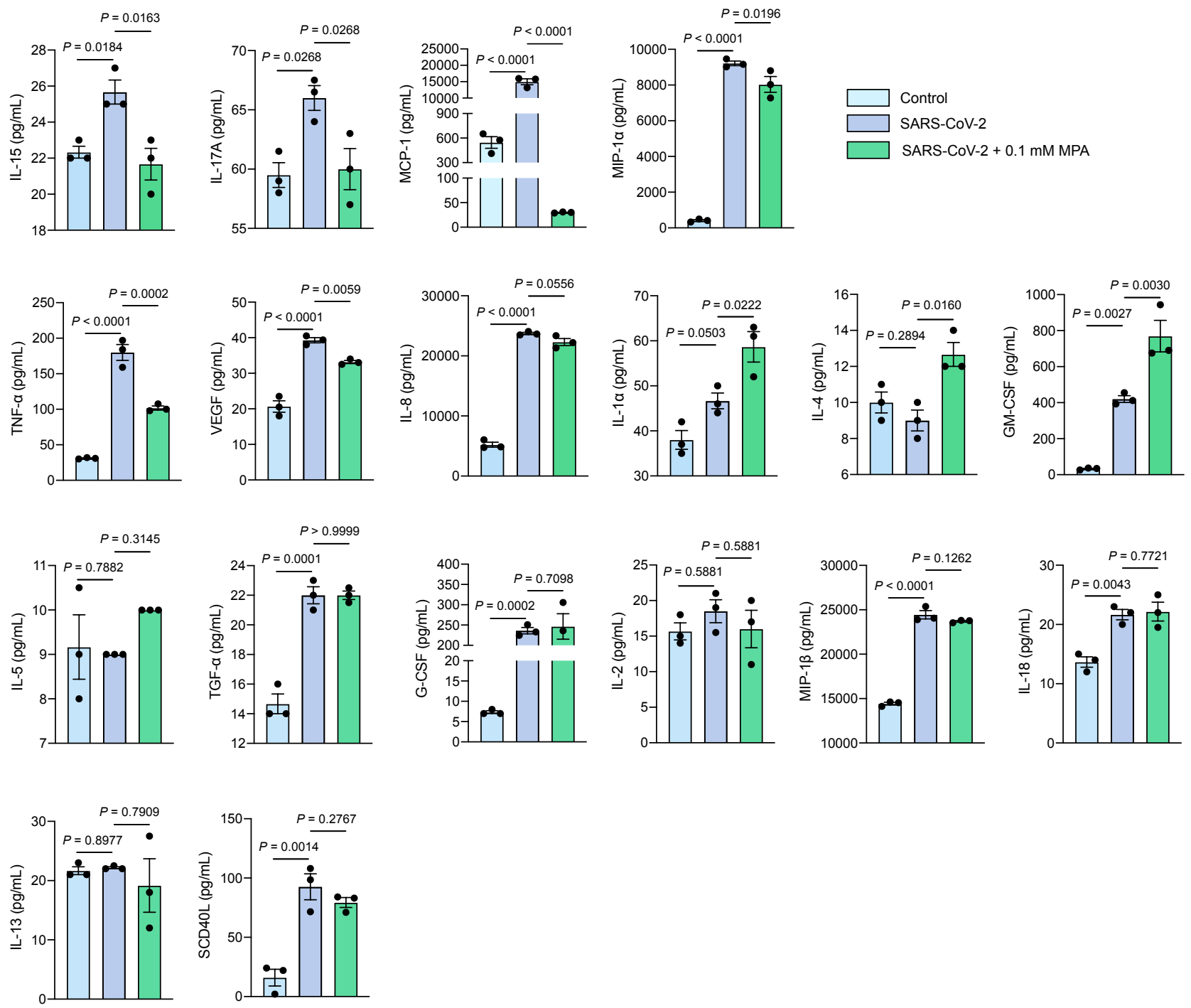


\section{Extended Data Figure 9}

a
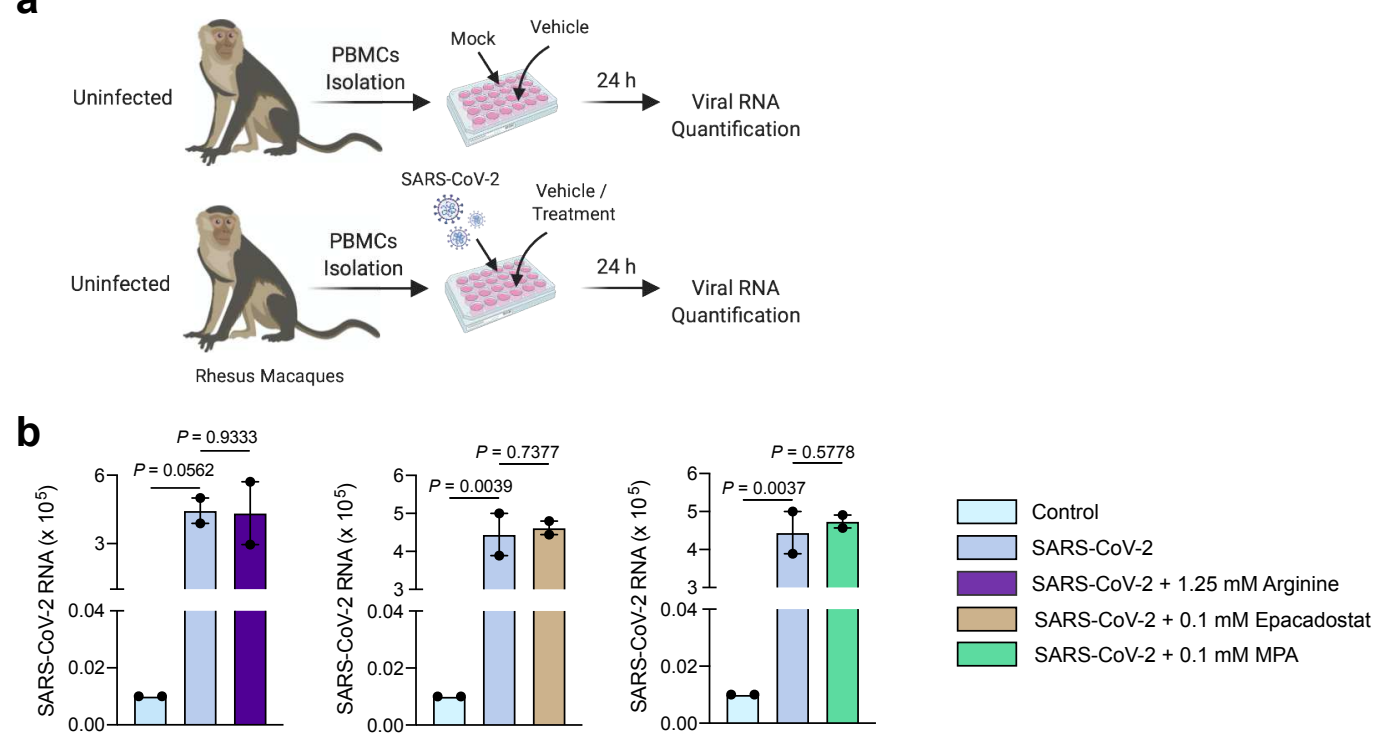


\section{Extended Data Table 1 | Clinical characteristics of healthy controls and COVID-19}

\section{patients}

\begin{tabular}{|c|c|c|c|c|}
\hline \multirow[b]{2}{*}{ Variables } & \multirow{2}{*}{$\begin{array}{c}\text { Healthy Controls } \\
\text { Healthy Controls }(\mathrm{n}=17)\end{array}$} & \multicolumn{3}{|c|}{ COVID-19 Patients } \\
\hline & & Mild $(n=14)$ & Severe $(\mathrm{n}=23)$ & Follow-up $(\mathrm{n}=7)$ \\
\hline \multicolumn{5}{|l|}{ Sex - no. ${ }^{a}(\%)$} \\
\hline Male & $8(47.1 \%)$ & $8(57.1 \%)$ & $9(39.1 \%)$ & $4(57.1 \%)$ \\
\hline Female & $9(52.9 \%)$ & $6(42.9 \%)$ & $14(60.9 \%)$ & $3(42.9 \%)$ \\
\hline \multicolumn{5}{|l|}{ Age - year } \\
\hline Mean \pm SD. ${ }^{b}$ & $47.5 \pm 13.8$ & $34.2 \pm 19.0$ & $60.8 \pm 16.7$ & $41.9 \pm 19.3$ \\
\hline Median (IQR) ${ }^{\mathrm{c}}$ & $50(40-54)$ & $38(20-48)$ & $65(52-78)$ & $46(28-56)$ \\
\hline Range & $20-72$ & $0.66-67$ & $31-80$ & $16-63$ \\
\hline \multicolumn{5}{|l|}{ BMI, $\mathbf{k g} / \mathrm{m}^{2}$} \\
\hline Median (IQR) & $25.7(24.7-27.4)$ & $23.4(20.7-26.1)$ & $24.5(23.0-27.2)$ & - \\
\hline \multicolumn{5}{|c|}{ Time from Onset to Admission, days } \\
\hline Median (IQR) & - & $6(4-8)$ & $7(5-8)$ & $2(1-3)$ \\
\hline \multicolumn{5}{|l|}{ Symptoms - no. (\%) } \\
\hline Fever & - & $11(78.6 \%)$ & $13(56.5 \%)$ & $5(71.4 \%)$ \\
\hline Fatigue & - & $1(7.1 \%)$ & $12(52.2 \%)$ & $2(28.6 \%)$ \\
\hline Dry cough & - & $8(57.1 \%)$ & $12(52.2 \%)$ & $3(42.9 \%)$ \\
\hline Inappetence & - & $3(21.4 \%)$ & $13(56.5 \%)$ & - \\
\hline Myalgia & - & $1(7.1 \%)$ & $3(13.0 \%)$ & $2(28.6 \%)$ \\
\hline Dyspnea & - & - & $9(39.1 \%)$ & - \\
\hline Expectoration & - & $2(14.3 \%)$ & $9(39.1 \%)$ & - \\
\hline Pharyngalgia & - & $2(14.3 \%)$ & $1(4.3 \%)$ & $1(14.3 \%)$ \\
\hline Diarrhea & - & $3(21.4 \%)$ & $2(8.7 \%)$ & - \\
\hline Nausea & - & - & $3(13.0 \%)$ & - \\
\hline Dizziness & - & - & $3(13.0 \%)$ & - \\
\hline Headache & - & $1(7.1 \%)$ & $2(8.7 \%)$ & - \\
\hline Abdominal pain & - & - & $2(8.7 \%)$ & - \\
\hline Chill & - & $1(7.1 \%)$ & $4(17.4 \%)$ & - \\
\hline Rhinorrhea & - & $3(21.4 \%)$ & $3(13.0 \%)$ & - \\
\hline Chest stuffiness & - & $3(21.4 \%)$ & $9(39.1 \%)$ & - \\
\hline Nasal congestion & - & $1(7.1 \%)$ & $2(8.7 \%)$ & - \\
\hline \multicolumn{5}{|l|}{ Comorbidity- no. (\%) } \\
\hline Hypertension & - & - & $3(13.0 \%)$ & $1(14.3 \%)$ \\
\hline
\end{tabular}




\begin{tabular}{|c|c|c|c|c|}
\hline Cardiovascular disease & - & - & $1(4.3 \%)$ & - \\
\hline Diabetes & - & - & $2(8.7 \%)$ & - \\
\hline COPD & - & - & $1(4.3 \%)$ & - \\
\hline Chronic liver disease & - & $1(7.1 \%)$ & $1(4.3 \%)$ & - \\
\hline \multicolumn{5}{|l|}{ Oxygenation Index - mmHg } \\
\hline Median (IQR) & - & $390(362-395)$ & $276(160-340)$ & $429(384-450)$ \\
\hline \multicolumn{5}{|l|}{ Treatment - no. (\%) } \\
\hline Kaletra & - & $13(92.9 \%)$ & $23(100.0 \%)$ & $7(100.0 \%)$ \\
\hline Oseltamivir & - & $1(7.1 \%)$ & $3(13.0 \%)$ & - \\
\hline Interferon therapy & - & $14(100.0 \%)$ & $23(100.0 \%)$ & $7(100.0 \%)$ \\
\hline Antibiotic therapy & - & $1(7.1 \%)$ & $18(78.3 \%)$ & $2(28.6 \%)$ \\
\hline Glucocorticoid & - & - & $10(43.5 \%)$ & $2(28.6 \%)$ \\
\hline Chinese medicine therapy & - & $3(21.4 \%)$ & $17(73.9 \%)$ & $7(100.0 \%)$ \\
\hline Oxygen therapy & - & - & $9(39.1 \%)$ & $7(100.0 \%)$ \\
\hline Mechanical ventilation & - & $13(92.9 \%)$ & $23(100.0 \%)$ & - \\
\hline \multicolumn{5}{|l|}{ Blood routine } \\
\hline White blood cell count, $\times 10^{9} / \mathrm{L}$ & - & - & $9.6(9.3-13.8)$ & $4.7(4.3-6.1)$ \\
\hline Red blood cell count, $\times 10^{9} / \mathrm{L}$ & - & - & $4.1(3.8-4.1)$ & $4.9(4.4-5.3)$ \\
\hline Neutrophils count, $\times 10^{9} / \mathrm{L}$ & - & $2.7(2.1-3.2)$ & $3.4(3.0-4.9)$ & $2.7(2.4-3.7)$ \\
\hline Lymphocytes count, $\times 10^{9} / \mathrm{L}$ & - & $1.5(1.2-2.1)$ & $0.8(0.6-1.1)$ & $1.2(1.1-1.4)$ \\
\hline Platelets count, $\times 10^{9} / \mathrm{L}$ & - & - & $259.0(163.0-356.0)$ & $179(158-185.5)$ \\
\hline Haemoglobin, g/L & - & $30.8(30.3-31.5)$ & $29.6(28.8-30.7)$ & - \\
\hline \multicolumn{5}{|l|}{ Blood biochemistry } \\
\hline Alanine aminotransferase, $\mathrm{U} / \mathrm{L}$ & $17.0(15.0-22.0)$ & $16.6(13.8-33.7)$ & $37.3(18.2-45.0)$ & $28.0(21.0-29.5)$ \\
\hline Aspartate aminotransferase, $\mathrm{U} / \mathrm{L}$ & $19.0(17.0-24.0)$ & $20.9(17.4-26.3)$ & $30.0(20.4-39.1)$ & $26.0(22.5-27.0)$ \\
\hline$\gamma$-glutamyl transferase, $\mathrm{U} / \mathrm{L}$ & $18.5(17.8-20.5)$ & $24.0(14.0-44.0)$ & $49.0(24.0-62.0)$ & $23.0(19.0-38.5)$ \\
\hline Total bilirubin, $\mu \mathrm{mol} / \mathrm{L}$ & $11.8(9.4-14.6)$ & $8.9(5.2-11.3)$ & $14.8(8.4-19.8)$ & $7.7(5.2-11.4)$ \\
\hline Direct bilirubin, $\mu \mathrm{mol} / \mathrm{L}$ & $1.4(1.0-2.2)$ & $4.5(3.0-5.0)$ & $5.4(4.1-12.9)$ & $2.1(1.3-2.7)$ \\
\hline Indirect bilirubin, $\mu \mathrm{mol} / \mathrm{L}$ & $7.2(5.8-8.5)$ & $4.3(2.5-6.4)$ & $6.3(5.1-7.6)$ & - \\
\hline Lactate dehydrogenase, U/L & - & $208.0(157.0-226.0)$ & $340.0(242.0-381.0)$ & $139.0(123.2-151.0)$ \\
\hline
\end{tabular}




\begin{tabular}{|l|l|l|l|l|}
\hline C-reactive protein, $\mathrm{mg} / \mathrm{L}$ & - & $2.5(1.2-7.6)$ & $67.3(14.2-121.6)$ & $1.2(0.8-8.2)$ \\
\hline Procalcitonin, $\mathrm{ng} / \mathrm{ml}$ & - & $0.032(0.031-0.043)$ & $0.078(0.051-0.154)$ & $0.050(0.050-0.070)$ \\
\hline Creatinine, $\mu \mathrm{mol} / \mathrm{L}$ & $54.0(43.0-59.0)$ & $70.0(63.0-74.0)$ & $61.0(50.0-64.0)$ & $63.0(53.0-82.0)$ \\
\hline Urea, $\mathrm{mmol} / \mathrm{L}$ & $5.3(4.4-6.1)$ & $3.5(3.2-4.4)$ & $3.8(2.8-5.2)$ & $5.0(3.6-5.4)$ \\
\hline Uric acid, $\mu \mathrm{mol} / \mathrm{L}$ & $326.0(264.0-373.0)$ & - & $151.5(106.0-232.5)$ & $269.0(225.5-389.5)$ \\
\hline Glucose, $\mathrm{mol} / \mathrm{L}$ & $5.0(4.9-5.5)$ & $5.5(4.9-5.8)$ & $6.9(5.4-11.8)$ & $7.3(5.5-8.3)$ \\
\hline Total protein, g/L & $72.0(70.2-73.8)$ & $67.1(64.5-68.5)$ & $62.6(59.2-66.7)$ & $70.3(64.3-73.5)$ \\
\hline Albumin, g/L & $45.1(43.3-46.5)$ & $40.8(38.8-41.6)$ & $32.9(31.4-36.7)$ & - \\
\hline Globulin, g/L & $27.0(24.1-28.4)$ & $26.0(24.0-29.0)$ & $30.0(26.0-32.0)$ & $26.3(25.6-27.8)$ \\
\hline Triglyceride, $\mathrm{mmol} / \mathrm{L}$ & $1.4(1.1-2.0)$ & - & $1.3(0.9-2.0)$ & $1.1(1.0-1.4)$ \\
\hline Total cholesterol, mmol/L & $4.9(4.5-5.9)$ & - & $3.5(3.0-3.8)$ & $4.2(3.8-4.4)$ \\
\hline High density lipoprotein, & $1.3(1.1-1.5)$ & - & $0.9(0.8-1.1)$ & $1.2(1.2-1.4)$ \\
\hline mmol/L & $3.0(2.4-3.5)$ & - & $2.2(1.8-2.3)$ & $2.2(1.8-2.2)$ \\
\hline Low density lipoprotein, $\mathrm{mmol} / \mathrm{L}$ & & & \\
\hline
\end{tabular}

${ }^{\mathrm{a}}$ no. (\%): number.

bSD: Standard Deviation.

${ }^{\mathrm{c}} \mathrm{IQR}$ : Interquartile range. 
a

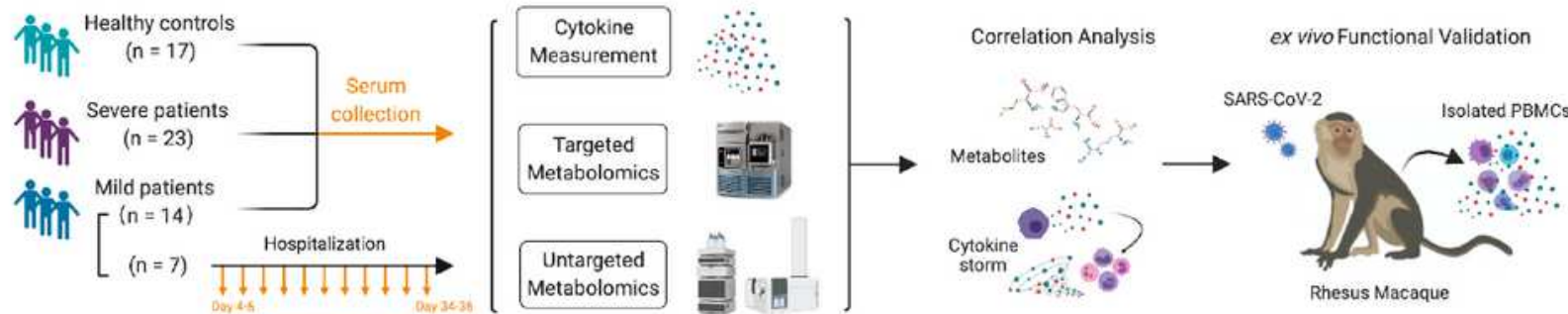

b

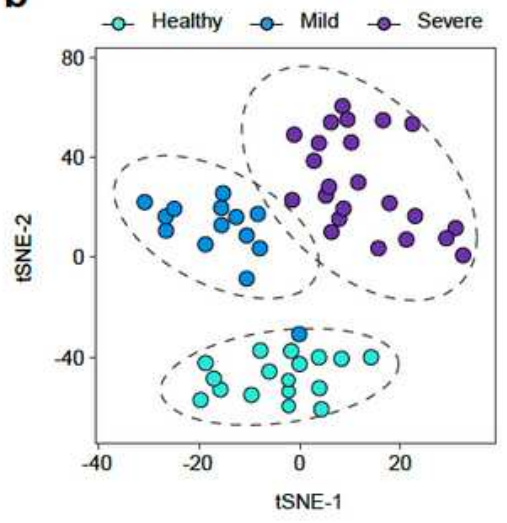

e

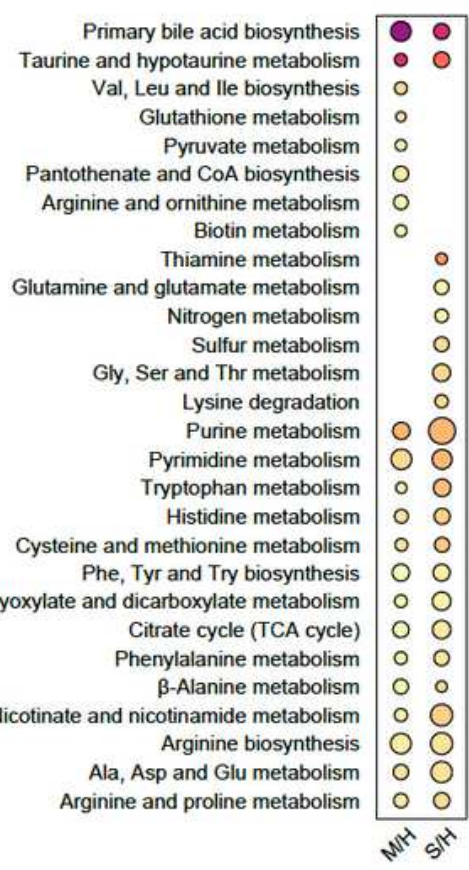

000
C

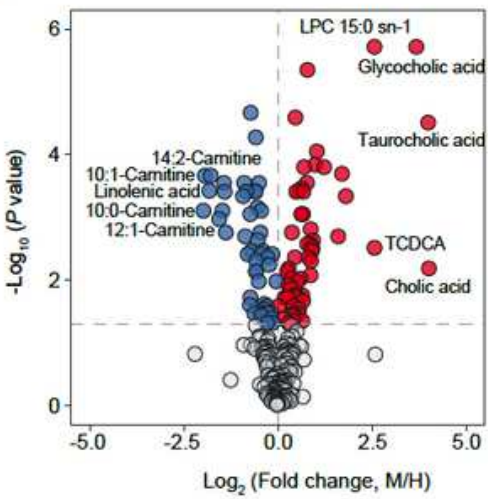

d

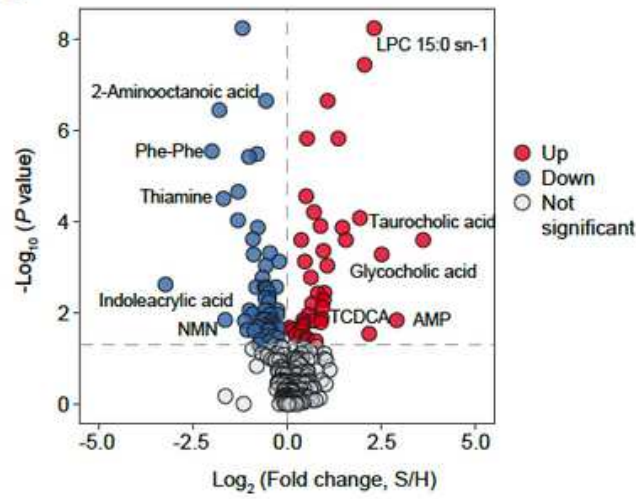

\section{f}
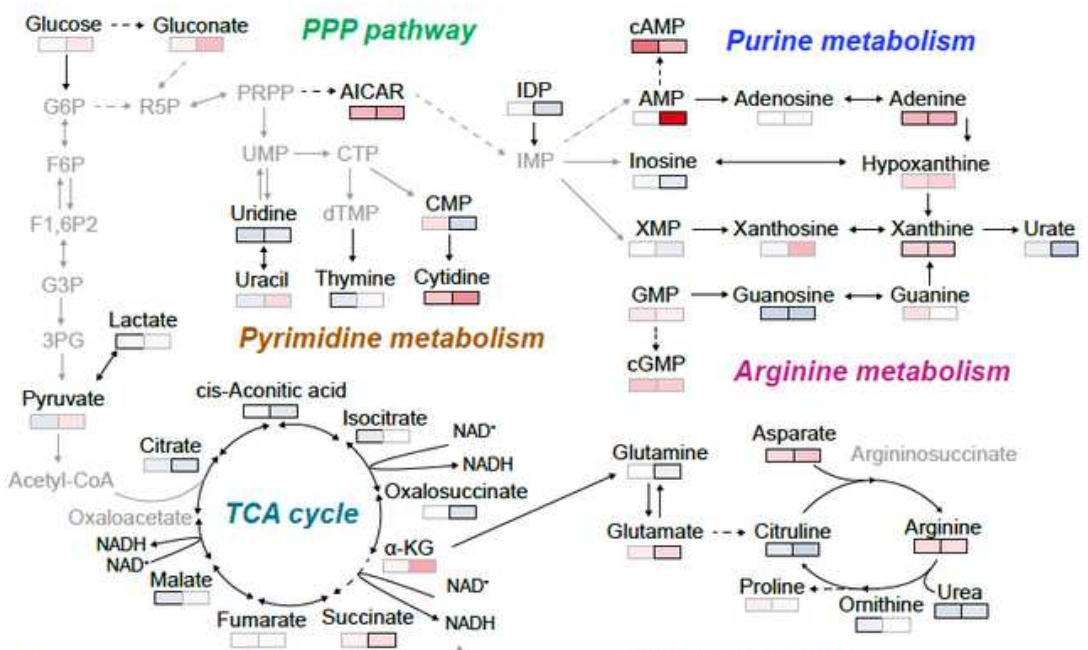

Tryptophan metabolism

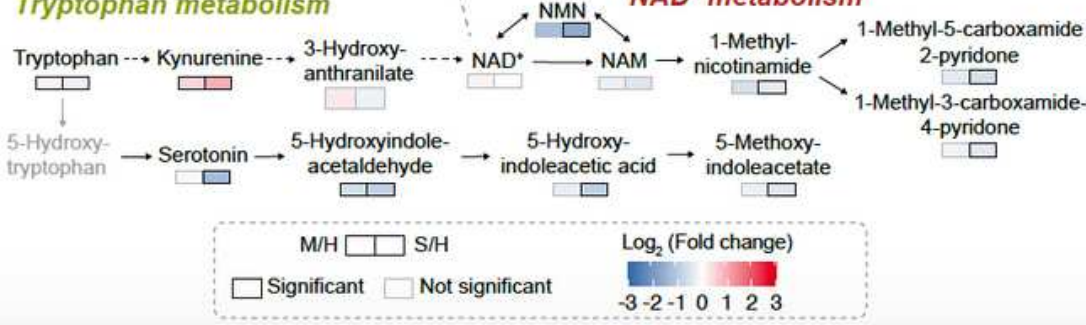

\section{Figure 1}

Study design and metabolic profiling in serum samples from mild and severe COVID-19 patients a, Overview of cohort (including mild $(n=21)$ and severe $(n=23)$ COVID-19 patients and healthy controls ( $n$ $=17)$ ) and the study design. $b, t-S N E$ plot distributed healthy controls $(n=17)$, mild patients $(n=14)$ and 
severe patients $(n=23)$ according to serum metabolites detected from targeted and untargeted metabolomics. c-d, Volcano plots comparing serum metabolites between mild (c) or severe (d) patients with healthy controls. Significantly increased and decreased metabolites are shown in red and blue, respectively, and metabolites with no statistical significance are shown in gray. Top 5 most significantly increased or decreased metabolites in each group are labeled. e, KEGG metabolic pathways in mild and severe patients enriched by significantly changed serum metabolites in $\mathrm{c}$ and $\mathrm{d}$. $\mathrm{f}$, Schematic depicting the key metabolic pathways altered in response to SARS-CoV-2 infection. Gray nodes represent metabolites that were not tested. Metabolite alteration are represented by color intensity, and borders are color-coded by statistical significance. Statistical significance was assessed using two-sided MannWhitney U test (c-d), and Fisher's exact test (e) followed by Benjamini-Hochberg multiple comparison test. 
a

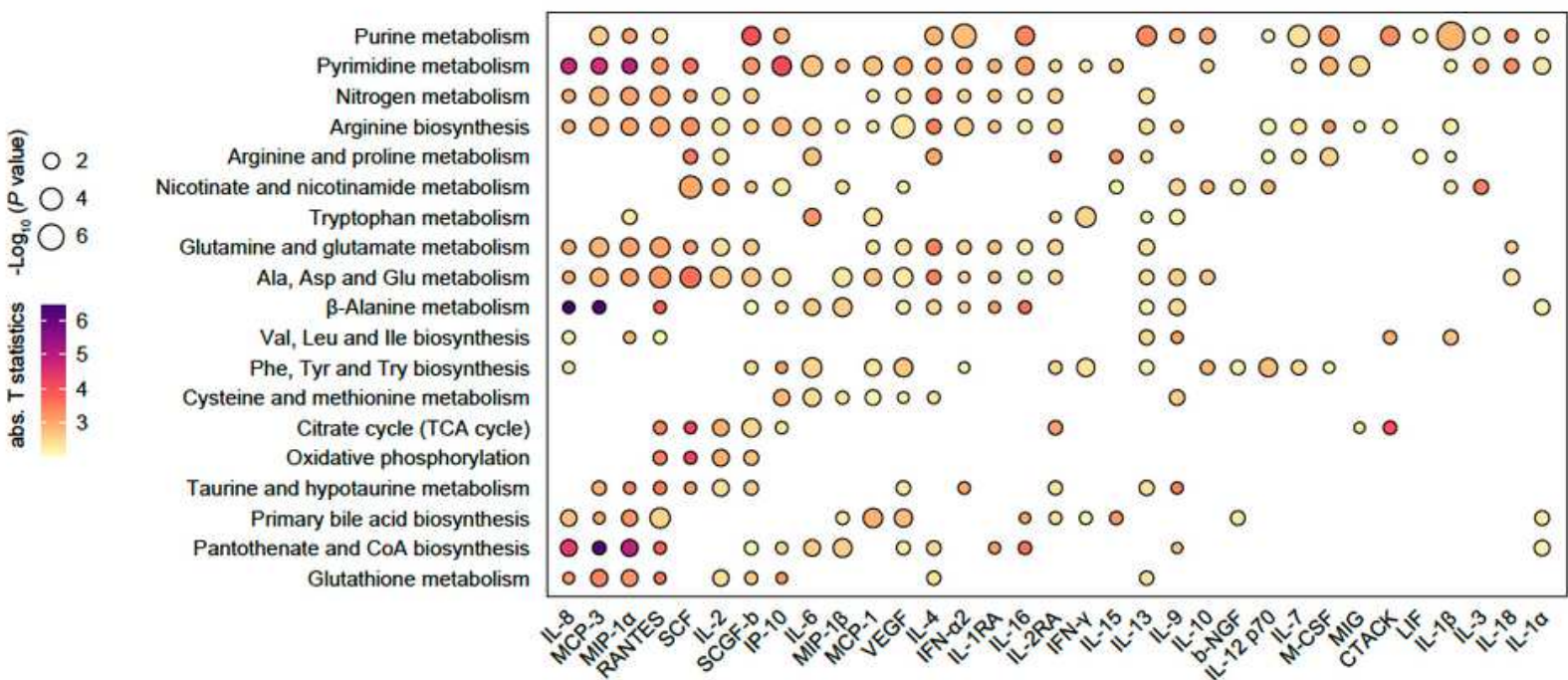
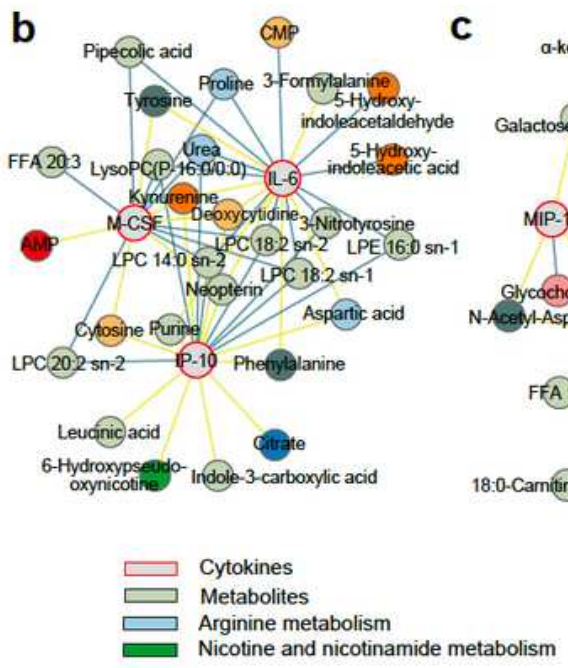

f

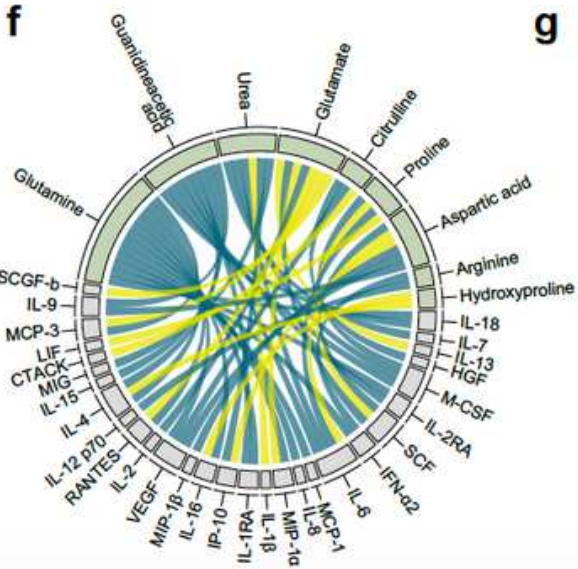

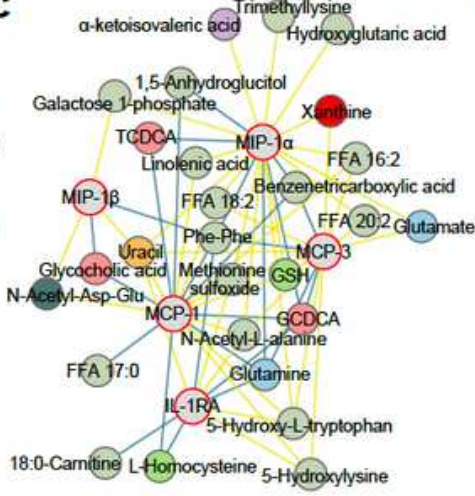

d

d $A 0$

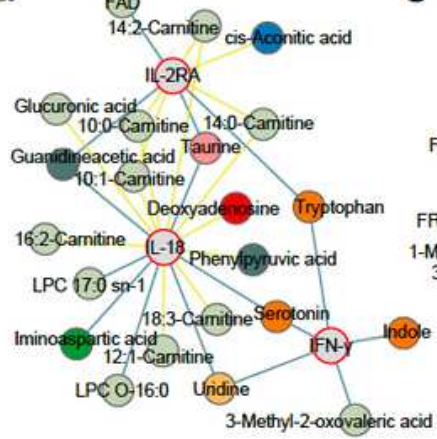

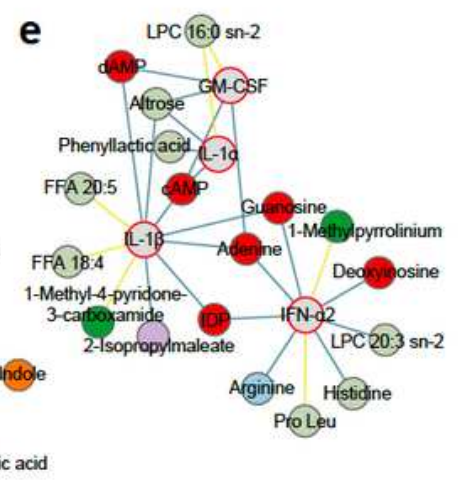

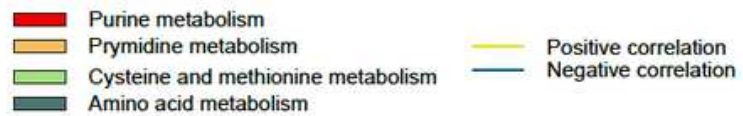
Tryptophan metabolism Val, Leu and lle metabolism
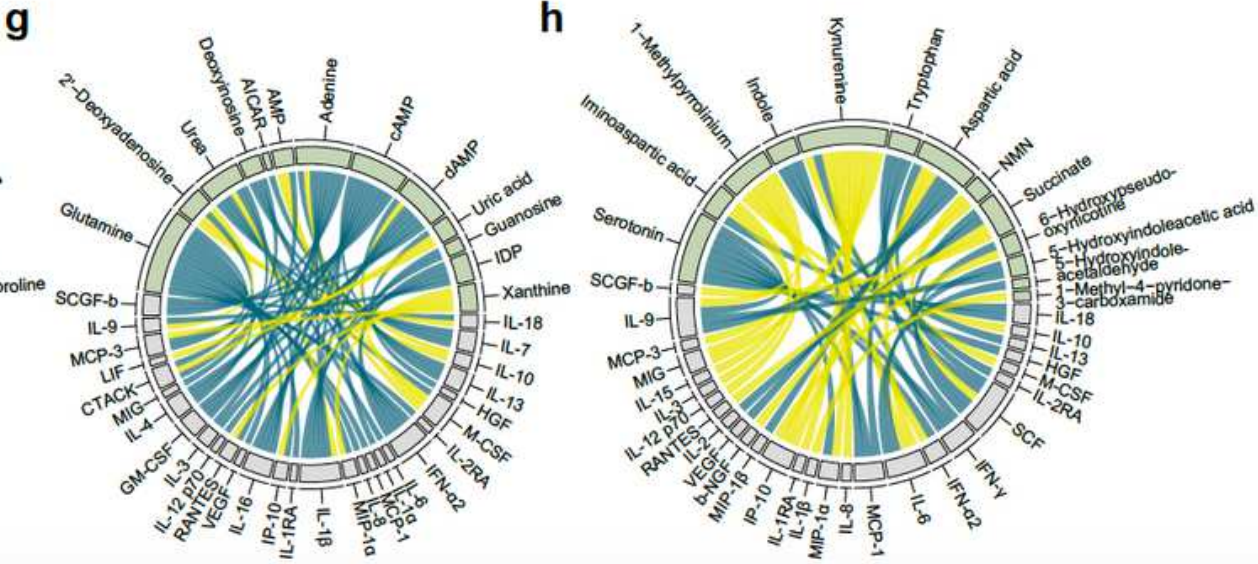

\section{Figure 2}

Metabolite-cytokine correlation in serum samples from COVID-19 patients a, Metabolic pathways enriched by metabolites that are significantly correlated with each presented cytokine. T statistics and statistical significance of metabolite-cytokine correlations were assessed based on two-sided t tests of regression coefficients followed by Benjamini-Hochberg multiple comparison test. Mean absolute (abs.) T statistics of metabolites in each pathway were calculated and represented by color intensity. Statistical 
significance of pathway enrichment analysis was assessed by Fisher's exact test and followed by Benjamini-Hochberg multiple comparison test. b-e, Correlation networks of key CRS-related cytokines and metabolites significantly altered in severe patients. Nodes are color-coded by molecule types and metabolic pathways. Edges are color-coded by association directions. Networks were clustered by fast greedy modularity optimization algorithm. $\mathrm{f}$-h, Chord diagrams of significant metabolite-cytokine correlations with metabolites involved in arginine metabolism (f), purine metabolism (g), tryptophan and NAD+ metabolism (h), respectively. Chords are color-coded by association directions consistent with b-e.

a

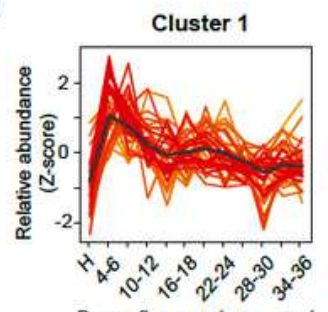

Days after symptoms onset

Cluster 3

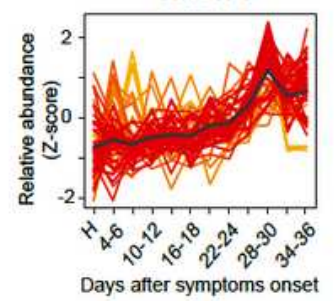

C

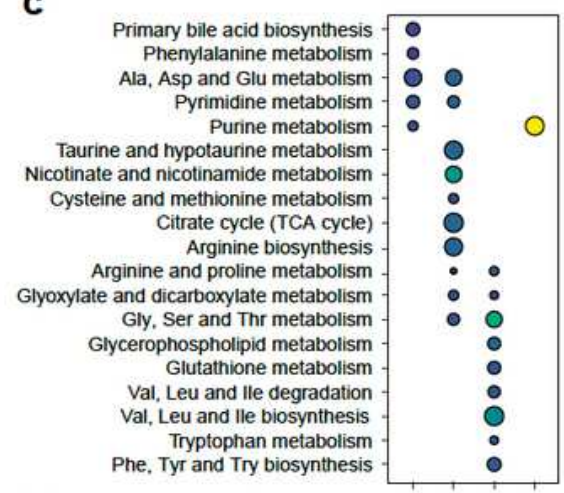

Fold enrichment $\quad \log _{10}(P$ value $)$

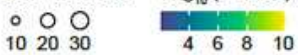

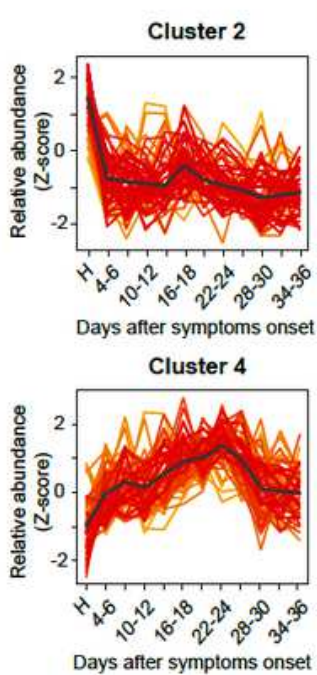

d
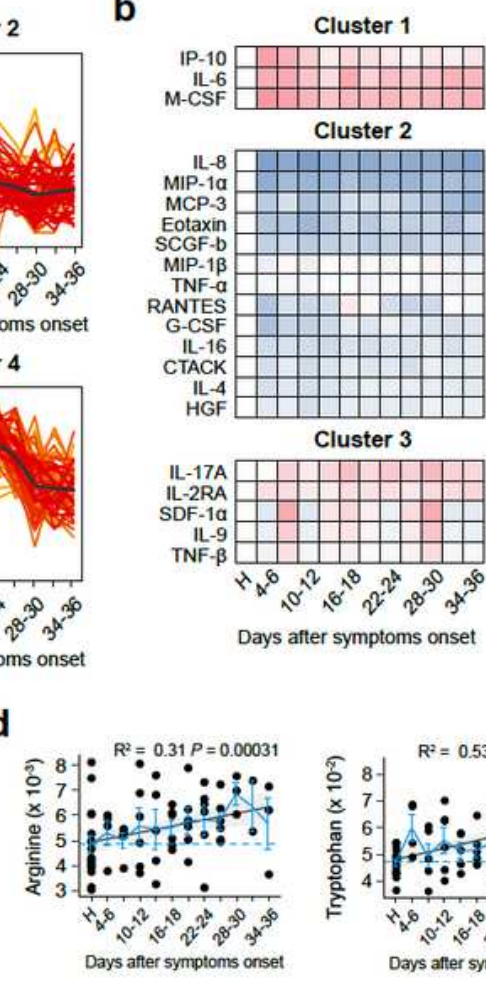

Cluster 3

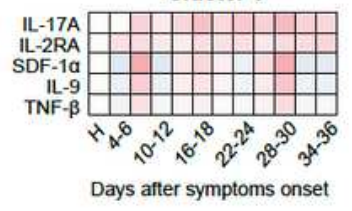

Cluster 4

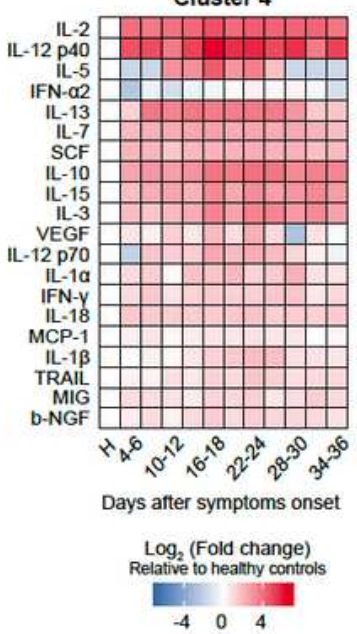

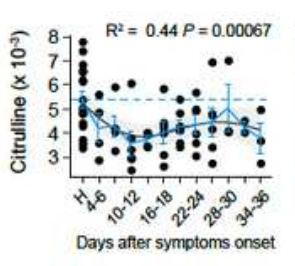
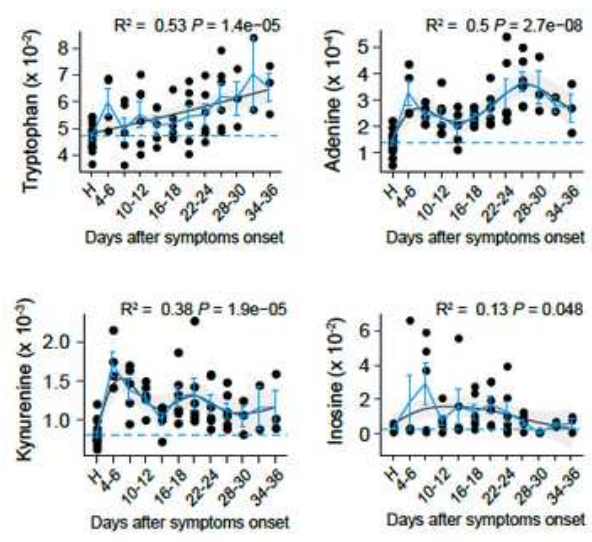

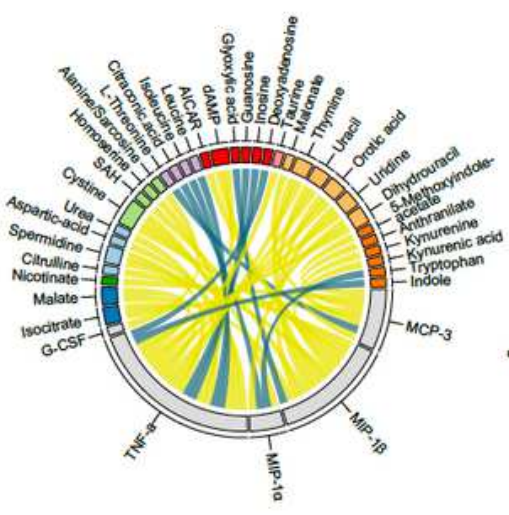

Primary bile acid biosynthesis
Citrate cycle (TCA cycle)
Tryptophan metabolism

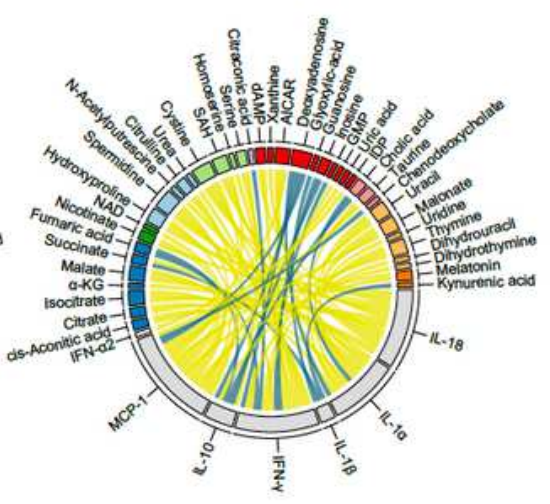

$\square$ Cytokines

$\square$ Arginine metabolism

Nicotine and nicotinamide metabolism

$\rightleftarrows$ Val, Leu and lie metabolism

Purine metabolism

$\square$ Prymidine metabolism

_ $\quad \begin{aligned} & \text { Positive correlation } \\ & \text { Negative correlation }\end{aligned}$ 


\section{Figure 3}

Longitudinal trajectories and metabolite-cytokine correlation in mild COVID- 19 patients a, Longitudinal trajectory clustering of significantly changed serum metabolites, cytokines in follow-up patients with mild COVID-19. Metabolite and cytokine abundance in healthy controls were used as base line. Black lines represent the average trajectory for each cluster. b, Heatmap comparison of cytokines at distinct time point in follow-up patients. Log2 fold change of mean cytokine abundance in each interval relative to healthy controls is represented by color intensity. c, Enriched KEGG metabolic pathways in each cluster. d, Serum metabolite trajectories based on normalized data in follow-up patients. Blue solid lines pass through the mean of each measurement at the specific time interval, and dotted lines represent the mean of measurements in healthy controls. Generalized addictive model (GAM) regression lines were represented by the black solid lines, with $95 \%$ confidence intervals for the regression line donated by gray filled areas. Data are presented as mean \pm SEM. with individual data points shown. e, Chord diagrams of significant associations between metabolites and core CRS-related cytokines in cluster 1 (left), cluster 2 (middle), cluster 3 and 4 (right), respectively. Statistical significance of was assessed by Fisher's exact test (c) and linear regression model adjusted for age and gender (e) followed by Benjiamini-Hochberg multiple comparison test. 
a
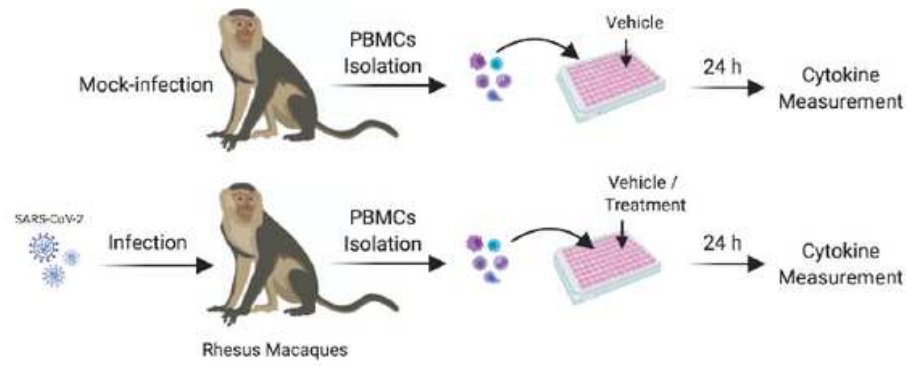

b
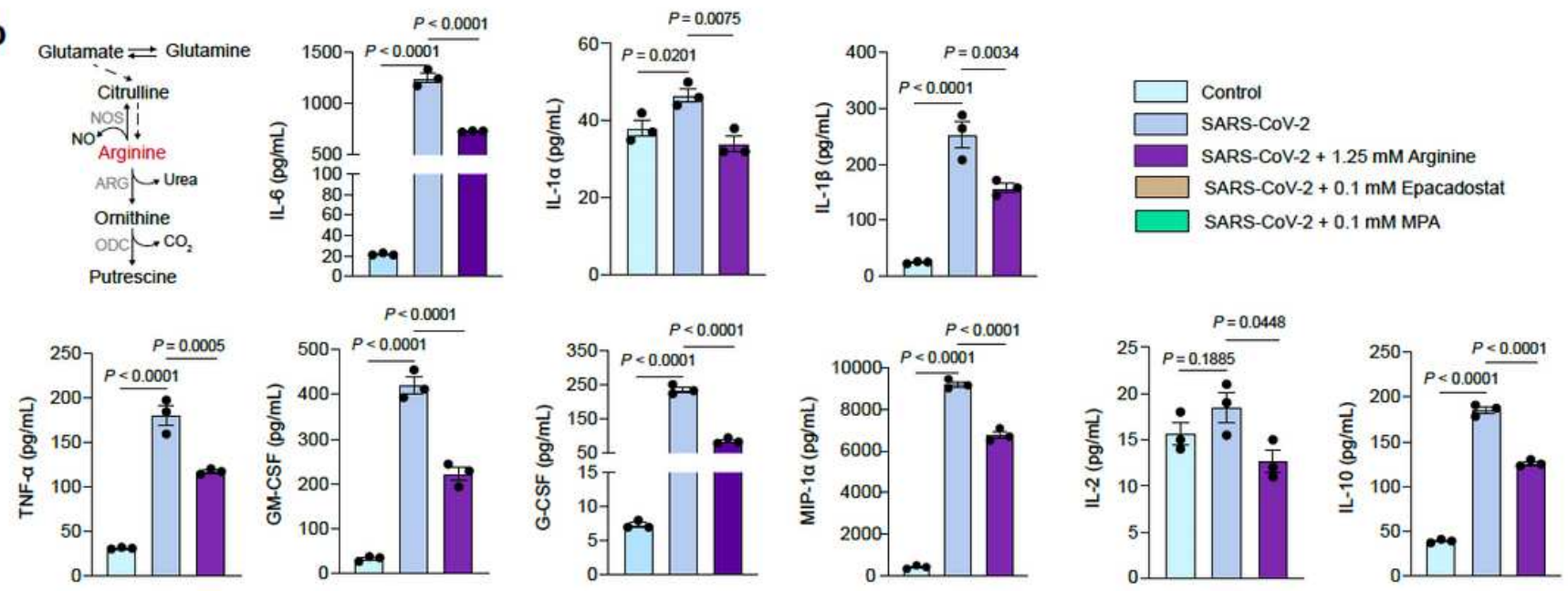

C
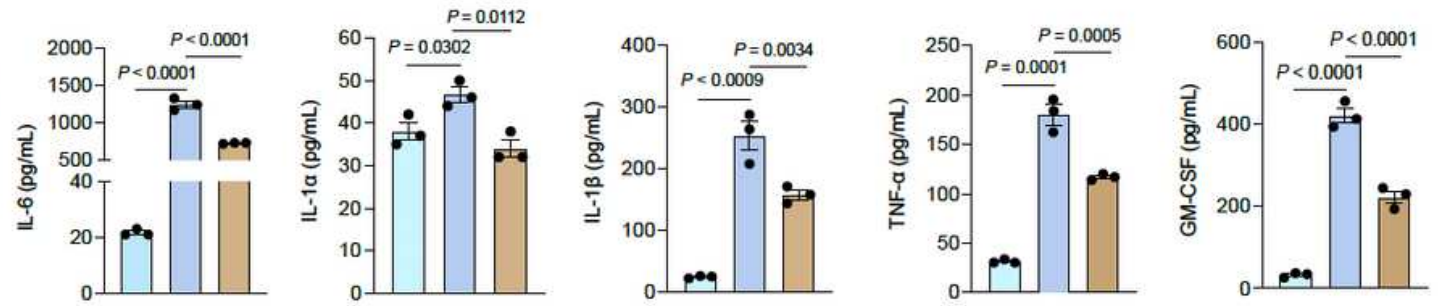

d
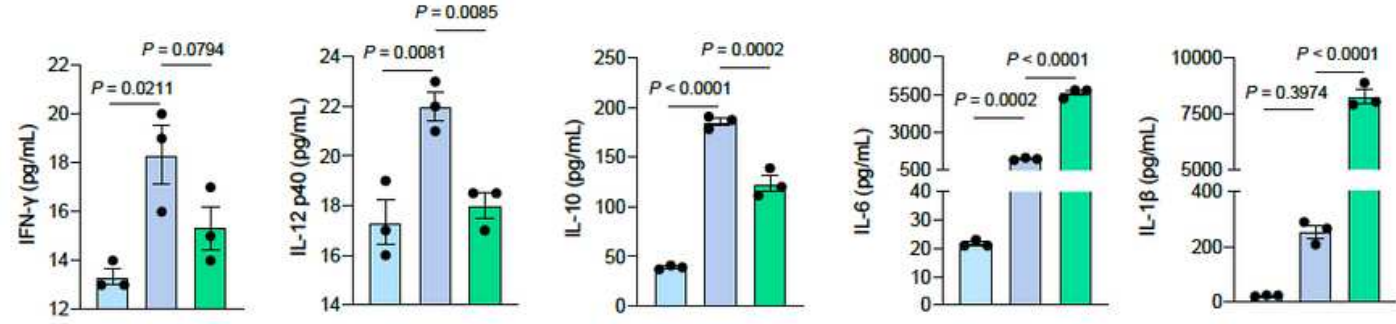

\section{Figure 4}

Targeting metabolism modulates cytokine release in PBMCs ex vivo model a, Schematic representation of the experimental workflow. PBMCs, isolated from peripheral blood of mock-infected or SARS-CoV-2infected rhesus macaques, were seeded in 96-well plates with vehicle or different drugs dissolved in the medium. 24 hours post seeding, cytokine abundance in cell culture was quantified. b-d, Metabolism diagrams and level of indicated cytokines and chemokines measured $24 \mathrm{~h}$ after supplementation of 1.25 $\mathrm{mM}$ arginine (b), $0.1 \mathrm{mM}$ ID01 inhibitor Epacadostat (c), and $0.1 \mathrm{mM}$ IMPDH inhibitor Mycophenolic acid $(M P A, d)$ in PBMCs. Data are presented as mean \pm SEM. with individual data points shown. Statistical 
significance was assessed using one-way ANOVA followed by Benjamini-Hochberg m 780 ultiple comparison test.

\section{Supplementary Files}

This is a list of supplementary files associated with this preprint. Click to download.

- Figs1.png

- Figs2.png

- FigS3.jpg

- FigS4.jpg

- FigS5.jpg

- Figs6.png

- FigS7.png

- Figs8.png

- Figs9.png 